MATHEMATICS OF COMPUTATION

Volume 73, Number 247, Pages 1393-1415

S 0025-5718(03)01595-3

Article electronically published on August 4, 2003

\title{
NUMERICAL SIMULATION \\ OF STOCHASTIC EVOLUTION EQUATIONS ASSOCIATED TO QUANTUM MARKOV SEMIGROUPS
}

\author{
CARLOS M. MORA
}

\begin{abstract}
We address the problem of approximating numerically the solutions $\left(X_{t}: t \in[0, T]\right)$ of stochastic evolution equations on Hilbert spaces $(\mathfrak{h},\langle\cdot, \cdot\rangle)$, with respect to Brownian motions, arising in the unraveling of backward quantum master equations. In particular, we study the computation of mean values of $\left\langle X_{t}, A X_{t}\right\rangle$, where $A$ is a linear operator. First, we introduce estimates on the behavior of $X_{t}$. Then we characterize the error induced by the substitution of $X_{t}$ with the solution $X_{t, n}$ of a convenient stochastic ordinary differential equation. It allows us to establish the rate of convergence of $\mathbf{E}\left\langle\tilde{X}_{t, n}, A \tilde{X}_{t, n}\right\rangle$ to $\mathbf{E}\left\langle X_{t}, A X_{t}\right\rangle$, where $\tilde{X}_{t, n}$ denotes the explicit Euler method. Finally, we consider an extrapolation method based on the Euler scheme. An application to the quantum harmonic oscillator system is included.
\end{abstract}

\section{INTRODUCTION}

This paper is concerned with the computation of $\mathbf{E}\left\langle X_{t}, A X_{t}\right\rangle$, where $A$ is a linear operator and $X_{t}$ satisfies the following linear stochastic evolution equation on the infinite dimensional separable (complex) Hilbert space $(\mathfrak{h},\langle\cdot, \cdot\rangle)$

$$
X_{t}=x_{0}+\int_{0}^{t} G X_{s} d s+\sum_{k=1}^{m} \int_{0}^{t} L_{k} X_{s} d W_{s}^{k}, \quad t \in[0, T],
$$

with $x_{0} \in \mathfrak{h},\left\{W^{k}\right\}_{k=1, \ldots, m}$ independent standard Brownian motions on a filtered complete probability space $\left(\Omega, F,\left(F_{t}\right)_{t \geq 0}, \mathbf{P}\right), T \in \mathbb{R}_{+}$and $m \in \mathbb{N}$. Here, all integrals are understood in the Itô sense and $L_{1}, \ldots, L_{m}, G$ are general linear operators in $\mathfrak{h}$. Furthermore, we will focus our attention on the case

$$
G=-i H-\frac{1}{2} \sum_{j=1}^{m} L_{j}^{*} L_{j}
$$

where $H$ is a self-adjoint operator in $\mathfrak{h}$.

Received by the editor August 25, 2002 and, in revised form, January 7, 2003.

2000 Mathematics Subject Classification. Primary 60H35; Secondary 60H10, 60H15, 60H30, $65 \mathrm{C} 30,65 \mathrm{C} 05$.

Key words and phrases. Stochastic evolution equation, numerical solution, rate of convergence, Euler scheme, Galerkin method, quantum dynamical semigroup, quantum master equation.

This research has been partially supported by FONDECYT grant 2000036, a DIPUC Ph.D. grant and the program "Cátedra Presidencial on Qualitative Analysis of Quantum Dynamical Systems". 
Our main motivation came from the numerical simulation of open quantum systems. In particular, we are interested in problems arising in quantum optics. Next, we give a brief introduction to this application. In the framework of quantum mechanics the state of a physical system is defined by specifying an element of an adequate complex Hilbert space [1, 4]. Since $\mathfrak{h}$ plays the role here of this quantum state space, $\mathfrak{h}$ describes, for example, one-particle wave functions [1, 4] or the Fock representation of the states in the second quantization formalism [1. In this context, $x_{0}$ is the initial state. Moreover, $\mathbf{E}\left\langle X_{t}, A X_{t}\right\rangle$ is interpreted as the mean value of the observable $A$ at the instant $t$ whenever $A$ is self-adjoint. This interpretation is easily obtained in the approach that describes the dynamics of quantum systems by stochastic evolutions of the corresponding state vectors [3 19. This technique allows us to characterize, for instance, the dynamics of an individual quantum system which is continuously monitored by some measurement device [14]. Alternatively, in the Heisenberg picture the evolution of the observable $A$, in systems whose initial state is $x_{0}$, is simulated by $\left\langle x_{0}, \Phi_{t}[A] x_{0}\right\rangle$, where $\Phi_{t}[A]$ is the minimal solution of the backward quantum master equation

$$
\begin{aligned}
\Phi_{0}[A] & =A, \quad \text { and } \forall \psi, \phi \in \mathfrak{D}=\operatorname{Dom}(G) \cap\left(\bigcap_{k=1}^{m} \operatorname{Dom}\left(L_{k}\right)\right), \\
\frac{d}{d t}\left\langle\psi, \Phi_{t}[A] \phi\right\rangle & =\left\langle G \psi, \Phi_{t}[A] \phi\right\rangle+\left\langle\psi, \Phi_{t}[A] G \phi\right\rangle+\sum_{j=1}^{m}\left\langle L_{j} \psi, \Phi_{t}[A] L_{j} \phi\right\rangle .
\end{aligned}
$$

Here $H$ represents the Hamiltonian and the operators $L_{j}, j=1, \ldots, m$, describe the effects of the environment. Now, we have that if $A$ is a bounded linear operator, then under weak conditions [10, 2

$$
\mathbf{E}\left\langle X_{t}, A X_{t}\right\rangle=\left\langle x_{0}, \Phi_{t}[A] x_{0}\right\rangle .
$$

We conclude this paragraph with some complementary remarks. First, the property (1.4) leads us to solve (1.3) through the weak numerical solution of (1.1). Furthermore, this procedure allows us to overcome the difficulties arising in the direct numerical integration of (1.3) in many cases; see, e.g., 19]. Second, $\Phi_{t}$ commonly define a minimal quantum dynamical semigroup on $B(\mathfrak{h})$ [7]. Third, (1.3) is based on the Born-Markov approximation of quantum phenomena, which has been used in quantum optics with great success. Finally, it is worth pointing out that there is a semigroup $\Psi_{t}$ in the space of trace-class operators such that $\Phi_{t}$ is the adjoint of $\Psi_{t}$ [7]; that is, $\Psi_{t}$ is the predual semigroup of $\Phi_{t}$. Hence

$$
\mathbf{E}\left\langle X_{t}, A X_{t}\right\rangle=\operatorname{Tr}\left(\Psi_{t}\left[P_{x_{0}}\right] A\right),
$$

where Tr means the trace and $P_{x_{0}}$ is the orthogonal projection of $\mathfrak{h}$ over the linear span of $x_{0}$. Furthermore, $\Psi_{t}$ solves the forward quantum master equation [10, 7 ] and represents the evolution of the density operators in the Schrödinger picture.

To compute $\mathbf{E}\left\langle X_{t}, A X_{t}\right\rangle$, we consider a sequence $\left(\mathfrak{h}_{n}\right)_{n}$ of finite dimensional Hilbert spaces such that

$$
\begin{aligned}
& \text { P1: } \mathfrak{h}_{n} \subset \operatorname{Dom}(H) \cap\left(\bigcap_{k=1}^{m} \operatorname{Dom}\left(L_{k}\right)\right) \cap\left(\bigcap_{k=1}^{m} \operatorname{Dom}\left(L_{k}^{*}\right)\right), \\
& \text { P2: } \forall x \in \mathfrak{h}, \exists x_{n} \in \mathfrak{h}_{n}, \text { such that } x_{n} \rightarrow_{n} x .
\end{aligned}
$$

Then we may proceed as follows: 
Step 1: $\mathbf{E}\left\langle X_{t}, A X_{t}\right\rangle$ is approximated by $\mathbf{E}\left\langle X_{t, n}, A X_{t, n}\right\rangle$, where $X_{t, n}$ is a continuous adapted stochastic process with values on $\mathfrak{h}_{n}$ given by

$$
X_{t, n}=P_{n} X_{0}+\int_{0}^{t} G_{n} X_{s, n} d s+\sum_{k=1}^{m} \int_{0}^{t} L_{k, n} X_{s, n} d W_{s}^{k},
$$

with $P_{n}: \mathfrak{h} \rightarrow \mathfrak{h}_{n}$ the orthogonal projection of $\mathfrak{h}$ over $\mathfrak{h}_{n}, L_{k, n}=P_{n} L_{k}$, whenever $k=1, \ldots, m$, and

$$
G_{n}=-i P_{n} H-\frac{1}{2} \sum_{j=1}^{m} P_{n} L_{j}^{*} P_{n} L_{j} .
$$

Step 2: $\mathbf{E}\left\langle X_{t, n}, A X_{t, n}\right\rangle$ is simulated numerically using weak schemes for stochastic differential equations (SDE's)

Before going on, we would like to say a few words in relation to the definition of $G_{n}$. Fagnola and Chebotarev [5, 7] proved that the minimal quantum semigroup is Markov, i.e., $\Phi_{t}[I]=I$, under rather general hypotheses. Therefore (1.1) is conservative; that is, $\mathbf{E}\left\|X_{t}\right\|^{2}=\left\|X_{0}\right\|^{2}$, in a wide class of applications. The approximations $X_{t, n}$ preserve this property if $G_{n}$ is chosen as in (1.6). This is not necessarily the case if $G_{n}=P_{n} G$. Notice that the fact that (1.5) is conservative allows us to construct efficient numerical schemes [17.

Our primary objective is to start providing the numerical simulation of quantum phenomena using stochastic evolution equations like (1.1) [9, 3, 19, 14, 22] with a theoretical treatment. Particularly, in this article we are interested in the theoretical understanding of Steps 1 and 2.

This paper is organized in six sections. Section 2 is devoted to introducing notation and preliminary results. In Section 3 we assume analogous hypotheses to those used by Fagnola and Chebotarev to obtain the uniqueness of the solution of (1.3). Let $C$ be a linear operator associated to (1.1) through these conditions. Then we estimate the quantities $\sup _{t \in[0, T]} \mathbf{E}\left\|C^{1 / 2} X_{t}\right\|^{2}$ and $\sup _{t \in[0, T]} \mathbf{E}\left\|C^{1 / 2} X_{t, n}\right\|^{2}$. These bounds, called "a priori" estimates on $X_{t}$ and $X_{t, n}$, will play an important role in the characterization of the speed of convergence the approximations introduced in Steps 1 and 2. Section 4 provides the rate of convergence of $\mathbf{E}\left\langle X_{t, n}, A X_{t, n}\right\rangle$ to $\mathbf{E}\left\langle X_{t}, A X_{t}\right\rangle$ whenever $t \in[0, T]$. In other words, we address the problem of estimating $\left|\mathbf{E}\left\langle X_{t}, A X_{t}\right\rangle-\mathbf{E}\left\langle X_{t, n}, A X_{t, n}\right\rangle\right|$. The primary goal of Section 5 is to study the rate of convergence of $\mathbf{E}\left\langle Z_{T_{j}^{M}, n}^{M}, A Z_{T_{j}^{M}, n}^{M}\right\rangle$ to $\mathbf{E}\left\langle X_{T_{j}^{M}}, A X_{T_{j}^{M}}\right\rangle$, where $M \in$ $\mathbb{N}, T_{j}^{M}=j T / M, j=0, \ldots, M$, and $Z_{\cdot, n}^{M}$ is the Euler approximation of (1.5)), i.e., $Z_{0, n}^{M}=X_{0, n}$ and for any $t \in\left[T_{j}^{M}, T_{j+1}^{M}\right]$,

$$
Z_{t, n}^{M}=Z_{T_{j}^{M}, n}^{M}+G_{n} Z_{T_{j}^{M}, n}^{M}\left(t-T_{j}^{M}\right)+\sum_{k=1}^{m} L_{k, n} Z_{T_{j}^{M}, n}^{M}\left(W_{t}^{k}-W_{T_{j}^{M}}^{k}\right) .
$$

This is the first step to approaching more complex numerical schemes, for example, those based in the computation of $X_{t, n} /\left\|X_{t, n}\right\|$. Finally, in Section 6 we apply our main results to an example of a quantum harmonic oscillator.

\section{Preliminaries}

2.1. Notation. In the following $\mathbb{Z}_{+}=\mathbb{N} \cup\{0\}$. Let $p \in \mathbb{N}$ and $m=\left(m^{j}\right)_{j=1, \ldots, p}$, $n=\left(n^{j}\right)_{j=1, \ldots, p} \in \mathbb{Z}^{p}$. Then $|n|=\sum_{k=1}^{p}\left|n^{j}\right|$. In addition, we write $n \geq m$ iff 
for any $j=1, \ldots, p, n^{j} \geq m^{j}$. Now we set $[n, m]=\left\{k \in \mathbb{Z}^{p}: k \geq n\right.$ and $\left.m \geq k\right\}$. Analogously, we define the partial orders $\leq,<,>$ and the boxes ]·[, ]·], [·[ With each $c \in \mathbb{Z}$ is associated the vector $\vec{c}$ in $\mathbb{Z}^{p}$ with all coordinates equal to $c$.

As usual in quantum mechanics, we consider that the scalar product $\langle\cdot, \cdot\rangle$ is linear with respect to the second variable and antilinear with respect to the first one. Let $B$ be a linear operator. We use the notation $\operatorname{Dom}(B)$ for the domain of $B$, and $B^{*}$ will be the adjoint of $B$. If $\Gamma$ is any logical statement, then we consider the Boolean function

$$
\mathbf{1}_{\Gamma}= \begin{cases}1, & \text { if } \Gamma \text { is true } \\ 0, & \text { if } \Gamma \text { is false. }\end{cases}
$$

For notational clarity we shall often suppress the explicit dependence of $T_{j}^{M}$ and $Z_{\cdot, n}^{M}$ on $M$. Moreover, the same symbol $K$ will denote various constants in the forthcoming sections. These are always assumed to be independent of $\mathfrak{h}_{n}$ and $M$ unless it is explicitly stated otherwise.

\subsection{Preliminary results. Suppose that}

H1: $G$ is the infinitesimal generator of a strongly continuous contraction semigroup in $\mathfrak{h}$,

H2: $\operatorname{Dom}(G) \subset \operatorname{Dom}\left(L_{k}\right)$, whenever $k=1, \ldots, m$,

H3: for each $k=1, \ldots, m, L_{k}$ is closable,

H4: there is a core $\mathfrak{D}^{*}$ for $G^{*}$ such that $\mathfrak{D}^{*} \subset \operatorname{Dom}\left(L_{k}^{*}\right), k=1, \ldots, m$.

Hence from [10] we have that there exists a unique (a.s.) adapted stochastic process $X$ such that

- $X$ is weakly continuous in probability,

- $\mathbf{E}\left\|X_{t}\right\|^{2} \leq\left\|X_{0}\right\|^{2}$

- for all $\phi \in \mathfrak{D}^{*}$ and $t \in[0, T]$,

$$
\left\langle\phi, X_{t}\right\rangle=\left\langle\phi, X_{0}\right\rangle+\int_{0}^{t}\left\langle G^{*} \phi, X_{s}\right\rangle d s+\sum_{k=1}^{m} \int_{0}^{t}\left\langle L_{k}^{*} \phi, X_{s}\right\rangle d W_{s}^{k} .
$$

In addition, (1.4) defines a quantum dynamical semigroup $\Phi_{t}$ in $B(\mathfrak{h})$, which is the minimal solution of (1.3) when $\mathfrak{D}^{*}$ is an invariant domain of $e^{G^{*} t}$.

2.3. Auxiliary lemmata. For completeness we state and prove the following two lemmata.

Lemma 2.1. Let $Y \in L^{2}(\mathbf{P} ; \mathfrak{h})=\left\{f: \Omega \rightarrow \mathfrak{h}, \quad \mathbf{E}\|f\|^{2}<+\infty\right\}$. Suppose that there is a self-adjoint positive operator $C$ such that $\sup _{\varepsilon>0} \mathbf{E}\left\|C_{\varepsilon}^{1 / 2} Y\right\|^{2}<+\infty$, where $C_{\varepsilon}=(I+\varepsilon C)^{-1} C(I+\varepsilon C)^{-1}$. Then $Y \in \operatorname{Dom}\left(C^{1 / 2}\right)$ a.s. and

$$
\mathbf{E}\left\|C^{1 / 2} Y\right\|^{2}<+\infty \text {. }
$$

Proof. First, by the Banach-Alaoglu theorem, there are $\varepsilon_{n} \rightarrow_{n} 0$ and $g \in L^{2}(\mathbf{P} ; \mathfrak{h})$ such that for any $f \in L^{2}(\mathbf{P} ; \mathfrak{h})$,

$$
\mathbf{E}\left\langle C_{\varepsilon(n)}^{1 / 2} Y, f\right\rangle \rightarrow{ }_{n} \mathbf{E}\langle g, f\rangle .
$$

Next, we consider a linear operator $M_{C^{1 / 2}}$ in $L^{2}(\mathbf{P} ; \mathfrak{h})$ with domain

$$
\left\{f \in L^{2}(\mathbf{P} ; \mathfrak{h}): \forall w \in \Omega, f(w) \in \operatorname{Dom}\left(C^{1 / 2}\right) \text { and } \mathbf{E}\left\|C^{1 / 2} f\right\|^{2}<+\infty\right\}
$$


such that $M_{C^{1 / 2}} f=C^{\frac{1}{2}} f$ whenever $f \in \operatorname{Dom}\left(M_{C^{1 / 2}}\right)$. Since $C^{1 / 2}$ is self-adjoint [12], from the properties of the resolvent of $C$ it follows that

$$
\mathbf{E}\left\langle Y, M_{C^{1 / 2}} f\right\rangle=\lim _{n} \mathbf{E}\left\langle C^{1 / 2}\left(I+\varepsilon_{n} C\right)^{-1} Y, f\right\rangle=\mathbf{E}\langle g, f\rangle .
$$

Therefore $Y \in \operatorname{Dom}\left(M_{C^{1 / 2}}^{*}\right)$.

Finally, the conclusion follows as in the proof of Lemma 1.1 of [8]. In fact, if $\left(\phi_{k}\right)_{k>1}$ is an orthonormal basis of $L^{2}(\mathbf{P} ; \mathbb{C})$, then there are $y_{k}, g_{k} \in \mathfrak{h}, k \geq 1$, such that $Y=\sum_{k \geq 1} y_{k} \phi_{k}$ and $g=\sum_{k \geq 1} g_{k} \phi_{k}$. It is worth pointing out that the above equalities are understood in the $L^{2}(\mathbf{P} ; \mathfrak{h})$ sense. From (2.2) we obtain that for any $v \in \operatorname{Dom}\left(C^{1 / 2}\right),\left\langle y_{k}, C^{1 / 2} v\right\rangle=\left\langle g_{k}, v\right\rangle$. Then $g_{k}=\left(C^{1 / 2}\right)^{*} y_{k}$. This implies that $C^{1 / 2} Y=\sum_{k \geq 1} g_{k} \phi_{k}$ a.s. and the required result follows.

Lemma 2.2. Let $\alpha, \psi$ and $\phi:[a, b] \rightarrow \mathbb{R}_{+}$be integrable functions. Assume that $\psi$ is continuous. If $\alpha$ has a nonnegative continuous derivative and for all $t \in[a, b]$,

$$
\phi(t) \leq \alpha(t)+\int_{a}^{t} \psi(s) \sqrt{\phi(s)} d s
$$

then $\phi(t) \leq\left(\frac{1}{2} \int_{a}^{t} \psi(s) d s+\sqrt{\alpha(t)}\right)^{2}$.

Proof. By (2.3) we have that for any $\varepsilon>0$ and $t \in[a, b]$

$$
\frac{1}{2} \frac{\dot{\alpha}(t)+\psi(t) \sqrt{\phi(t)}}{\left(\varepsilon+\alpha(t)+\int_{a}^{t} \psi(s) \sqrt{\phi(s)} d s\right)^{1 / 2}} \leq \frac{1}{2} \frac{\dot{\alpha}(t)}{(\varepsilon+\alpha(t))^{1 / 2}}+\frac{\psi(t)}{2} .
$$

It follows that $\sqrt{\varepsilon+\phi(t)} \leq \frac{1}{2} \int_{a}^{t} \psi(s) d s+\sqrt{\varepsilon+\alpha(t)}$ and the proof is finished taking the limit as $\varepsilon \rightarrow 0$.

\section{A priori estimates on $X$ And $X_{\cdot, n}$}

This section has two main objectives: first, to obtain uniform estimates with respect to $n$ on the behavior of $X_{t, n}$; second, to state regularity results for $X_{t}$, where $X$ is the stochastic process $X$ given in subsection 2.2 .

Theorem 3.1. Suppose that assumptions $\mathrm{H}_{1}$ to $\mathrm{H}_{4}$ hold and

H5: for any $\phi \in \mathfrak{D}^{*}, G^{*} \phi=\lim _{n} G_{n}^{*} \phi$ and $L_{k}^{*} \phi=\lim _{n} L_{k, n}^{*} \phi$,

H6: $\forall n \in \mathbb{N}, \mathfrak{h}_{n} \subset \mathfrak{D}^{*}$,

H7: there is a self-adjoint positive operator $C$ such that for each $n \geq 0$, $\mathfrak{h}_{n} \subset \operatorname{Dom}\left(C^{1 / 2}\right)$ and $\forall \phi \in \mathfrak{h}_{n}$,

$$
2 \operatorname{Re}\left\langle C^{1 / 2} \phi, C^{1 / 2} G_{n} \phi\right\rangle+\sum_{k=1}^{m}\left\|C^{1 / 2} L_{k, n} \phi\right\|^{2} \leq K\left(\left\|C^{1 / 2} \phi\right\|^{2}+\|\phi\|^{2}\right),
$$

H8: $\forall \phi \in \operatorname{Dom}\left(C^{1 / 2}\right),\left\|C^{1 / 2} P_{n} \phi\right\| \rightarrow_{n \rightarrow+\infty}\left\|C^{1 / 2} \phi\right\|$.

If $X_{0} \in \operatorname{Dom}\left(C^{1 / 2}\right)$ a.s., then $X_{t} \in \operatorname{Dom}\left(C^{1 / 2}\right)$ a.s. and there is a constant $K$ such that

$$
\sup _{t \in[0, T]} \mathbf{E}\left\|C^{1 / 2} X_{t}\right\|^{2} \leq K\left(\left\|C^{1 / 2} X_{0}\right\|^{2}+\left\|X_{0}\right\|^{2}\right)
$$


and

$$
\sup _{t \in[0, T]} \mathbf{E}\left\|C^{1 / 2} X_{t, n}\right\|^{2} \leq K\left(\left\|C^{1 / 2} P_{n} X_{0}\right\|^{2}+\left\|X_{0}\right\|^{2}\right), \quad \text { for all } n \in \mathbb{N} .
$$

In a wide class of applications assumptions $\mathrm{H} 1$ to $\mathrm{H} 8$ hold. In fact, these conditions are analogous to those used in [7] to derive that there is a unique $\sigma$-weak continuous family $\left(\Phi_{t}\right)_{t \geq 0}$ of positive maps on $B(\mathfrak{h})$ satisfying (1.3).

Notice that the first stage of the Galerkin method [15 is fulfilled in Step 1. Now, we carry out the next three stages of this procedure in the proof of Theorem 3.1 Stage 4 rests on modifications of ideas presented in 8 .

Proof of Theorem 3.1. In the second stage of the Galerkin method we have to establish estimates on $X_{n}$. Here, since for any $\varphi \in \mathfrak{h}_{n}, 2 \operatorname{Re}\left\langle\varphi, G_{n} \varphi\right\rangle+\sum_{k=1}^{m}\left\|L_{k, n} \varphi\right\|^{2}=$ 0 , it follows from Itô's formula that

$$
\mathbf{E}\left\|X_{t, n}\right\|^{2}=\left\|X_{0, n}\right\|^{2} .
$$

In addition, for any $\phi \in \mathfrak{D}^{*}$,

$$
\left\langle\phi, X_{t, n}\right\rangle=\left\langle\phi, X_{0, n}\right\rangle+\int_{0}^{t}\left\langle G_{n}^{*} \phi, X_{s, n}\right\rangle d s+\sum_{k=1}^{m} \int_{0}^{t}\left\langle L_{k, n}^{*} \phi, X_{s, n}\right\rangle d W_{s}^{k} .
$$

Therefore, using (3.2), H5 and the integration by parts formula [20, we obtain that for all $\phi \in \mathfrak{D}^{*}$ there exists a constant $K_{\phi}$ such that

$$
\mathbf{E}\left|\left\langle\phi, X_{t, n}-X_{s, n}\right\rangle\right|^{2} \leq K_{\phi}(t-s)\left\|X_{0}\right\|^{2} .
$$

Finally, $C^{1 / 2} P_{n} \in B(\mathfrak{h})$ leads to

$$
C^{1 / 2} X_{t, n}=C^{1 / 2} X_{0, n}+\int_{0}^{t} C^{1 / 2} P_{n} G_{n} X_{s, n} d s+\sum_{k=1}^{m} \int_{0}^{t} C^{1 / 2} P_{n} L_{k, n} X_{s, n} d W_{s}^{k}
$$

Therefore for each $t \in[0, T]$,

$$
\begin{gathered}
\mathbf{E}\left\|C^{1 / 2} X_{t, n}\right\|^{2}=\mathbf{E}\left\|C^{1 / 2} X_{0, n}\right\|^{2}+2 \mathbf{E} \int_{0}^{t} \operatorname{Re}\left\langle C^{1 / 2} X_{s, n}, C^{1 / 2} G_{n} X_{s, n}\right\rangle d s \\
+\sum_{k=1}^{m} \mathbf{E} \int_{0}^{t}\left\|C^{1 / 2} L_{k, n} X_{s, n}\right\|^{2} d s .
\end{gathered}
$$

Hence, by (3.2) and condition $\mathrm{H} 7$

$$
\mathbf{E}\left\|C^{1 / 2} X_{t, n}\right\|^{2} \leq \mathbf{E}\left\|C^{1 / 2} X_{0, n}\right\|^{2}+K t\left\|X_{0}\right\|^{2}+\int_{0}^{t} \mathbf{E}\left\|C^{1 / 2} X_{s, n}\right\|^{2} d s .
$$

This implies

$$
\mathbf{E}\left\|C^{1 / 2} X_{t, n}\right\|^{2} \leq K\left(\left\|C^{1 / 2} X_{0, n}\right\|^{2}+\left\|X_{0}\right\|^{2}\right) .
$$

Next, we consider the third stage of the Galerkin method, where we have to study the limit as $k \rightarrow \infty$ of a certain subsequence $\left(X_{\cdot, n_{k}}\right)_{k}$.

Let $\tilde{F}_{t}$ be the $\sigma$-algebra generated by $\left(W_{s}\right)_{s \in[0, t]}$ and the $\mathbf{P}$-null sets of $F$. Thus, for each $n$ there is a $\left(\tilde{F}_{t}\right)_{t \geq 0}$-adapted stochastic process $\tilde{X}_{\cdot, n}$ such that $\tilde{X}_{\cdot, n}$ and 
$X_{\cdot, n_{k}}$ are indistinguishable. Now, by $\left(\underline{3.3)}\right.$ we can ensure that for all $\xi \in L^{2}(\mathbf{P} ; \mathbb{C})$ and $\phi \in \mathfrak{D}^{*}$,

$$
\left|\mathbf{E} \xi\left\langle\phi, \tilde{X}_{t, n}-\tilde{X}_{s, n}\right\rangle\right|^{2} \leq K_{\phi}(t-s)\left\|x_{0}\right\|^{2} \mathbf{E}|\xi|^{2} .
$$

Thus $\left\{\mathbf{E} \xi\left\langle\phi, \tilde{X}_{\cdot, n}\right\rangle\right\}_{n \geq 0}$ is a uniformly bounded and equicontinuous family of functions defined on $[0, T]$. Let $\left(\xi_{l} \phi_{l}\right)_{l \geq 1}$ be the sequence obtained by arranging all products of elements of the dense subset $\left\{\phi_{n}\right\}_{n \geq 1}$ of $\mathfrak{D}^{*}$ with elements of the dense subset $\left\{\xi_{n}\right\}_{n \geq 1}$ of $L^{2}(\mathbf{P} ; \mathbb{C})$. Hence Arzela-Ascoli's theorem and diagonalization arguments show that there exist a subsequence $\left(\tilde{X}_{\cdot, n_{k}}\right)_{k \geq 1}$ and a sequence $\left(f_{l}\right)_{l \geq 1}$ of continuous functions defined on $[0, T]$ such that $\mathbf{E} \xi_{l}\left\langle\phi_{l}, \tilde{X}_{\cdot, n_{k}}\right\rangle \rightrightarrows_{k} f_{l}$ and $\left\|\mathbf{E} \xi_{l}\left\langle\phi_{l}, \tilde{X}_{\cdot, n_{k}}\right\rangle-f_{l}\right\|_{\infty}<1 / l$, whenever $k \geq l$.

On the other hand, using (3.2), we obtain that for each $t \in[0, T]$ there is $\bar{X}_{t} \in$ $L^{2}\left(\mathbf{P}, \tilde{F}_{t} ; \mathfrak{h}\right)$ and a subsequence $\left(\tilde{X}_{t, n_{k(p)}}\right)_{p \geq 0}$, such that

$$
\tilde{X}_{t, n_{k(p)}} \longrightarrow{ }_{p \rightarrow+\infty}^{\text {weak }^{*}} \bar{X}_{t}
$$

Then $f_{l}(t)=\mathbf{E} \xi_{l}\left\langle\phi_{l}, \bar{X}_{t}\right\rangle$ and

$$
\mathbf{E}\left\|\bar{X}_{t}\right\|^{2} \leq \liminf \mathbf{E}\left\|\tilde{X}_{t, n_{k(p)}}\right\|^{2} \leq\left\|x_{0}\right\|^{2} .
$$

Therefore it follows from the previous paragraph that for each $Z \in L^{2}(\mathbf{P} ; \mathfrak{h})$, $\mathbf{E}\left\langle Z, \tilde{X}_{\cdot, n_{k}}\right\rangle \rightrightarrows_{k \rightarrow+\infty} \mathbf{E}\langle Z, \bar{X}\rangle$.

We are ready to proceed with Stage 4 of the Galerkin method, which concerns the properties of $\bar{X}$.

Let $\xi \in L^{2}\left(\mathbf{P}, \tilde{F}_{t} ; \mathbb{C}\right)$. Then there exist predictable processes $H^{k}, k=1, \ldots, m$, in $L^{2}([0, t] \times \Omega ; v \times \mathbf{P} ; \mathbb{C})$ such that $\xi=\mathbf{E} \xi+\sum_{k=1}^{m} \int_{0}^{t} H_{s}^{k} d W_{s}^{k}$. Thus we have that for any $\phi \in \mathfrak{D}^{*}$ and $j=1, \ldots, m$,

$$
\begin{gathered}
\mathbf{E} \xi \int_{0}^{t}\left\langle L_{j, n_{k}}^{*} \phi, \tilde{X}_{s, n_{k}}\right\rangle d W_{s}^{j}-\mathbf{E} \xi \int_{0}^{t}\left\langle L_{j, n_{k}}^{*} \phi, \bar{X}_{s}\right\rangle d W_{s}^{j} \\
=\mathbf{E} \int_{0}^{t} H_{s}^{l}\left(\left\langle L_{j, n_{k}}^{*} \phi, \tilde{X}_{s, n_{k}}\right\rangle-\left\langle L_{j, n_{k}}^{*} \phi, \bar{X}_{s}\right\rangle\right) d s .
\end{gathered}
$$

As a consequence, taking the limit as $k \rightarrow \infty$ in

$$
\begin{aligned}
\mathbf{E} \xi\left\langle\phi, \tilde{X}_{t, n_{k}}\right\rangle=\mathbf{E} \xi\left\langle\phi, \tilde{X}_{0, n_{k}}\right\rangle+\mathbf{E} \int_{0}^{t} \xi\left\langle G_{n_{k}}^{*} \phi, \tilde{X}_{s, n_{k}}\right\rangle d s \\
+\sum_{j=1}^{m} \mathbf{E} \xi \int_{0}^{t}\left\langle L_{j, n_{k}}^{*} \phi, \tilde{X}_{s, n_{k}}\right\rangle d W_{s}^{j}
\end{aligned}
$$

we obtain

$$
\mathbf{E} \xi\left\langle\phi, \bar{X}_{t}\right\rangle=\mathbf{E} \xi\left\langle\phi, \bar{X}_{0}\right\rangle+\mathbf{E} \int_{0}^{t} \xi\left\langle G^{*} \phi, \bar{X}_{s}\right\rangle d s+\sum_{j=1}^{m} \mathbf{E} \xi \int_{0}^{t}\left\langle L_{j}^{*} \phi, \bar{X}_{s}\right\rangle d W_{s}^{j} .
$$

Then $\bar{X}$ satisfies (2.1). Since $\bar{X}$ is weakly continuous in the mean-square sense, it follows from subsection 2.2 that $\bar{X}=X$ a.s. 
Inspired by the proof of Theorem 2.2 of [8], we now consider the variant of the Yosida approximation $C_{\varepsilon}=(I+\varepsilon C)^{-1} C(I+\varepsilon C)^{-1}$. Combining (3.5) and $C_{\varepsilon}^{1 / 2} \leq C^{1 / 2}$, we arrive at

$$
\mathbf{E}\left\|C_{\varepsilon}^{1 / 2} X_{t, n}\right\|^{2} \leq K\left(\left\|C^{1 / 2} X_{0, n}\right\|^{2}+\left\|X_{0}\right\|^{2}\right) .
$$

On the other hand, for any $Z \in L^{2}(\mathbf{P} ; \mathfrak{h})$ and each $t \in[0, T]$

$$
\mathbf{E}\left\langle Z, C_{\varepsilon}^{1 / 2} X_{t, n_{k}}\right\rangle=\mathbf{E}\left\langle C_{\varepsilon}^{1 / 2} Z, X_{t, n_{k}}\right\rangle \rightarrow_{k} \mathbf{E}\left\langle Z, C_{\varepsilon}^{1 / 2} X_{t}\right\rangle .
$$

Then (3.6) leads to

$$
\mathbf{E}\left\|C_{\varepsilon}^{1 / 2} X_{t}\right\|^{2} \leq \liminf _{k} \mathbf{E}\left\|C_{\varepsilon}^{1 / 2} X_{t, n_{k}}\right\|^{2} \leq K\left(\left\|C^{1 / 2} X_{0}\right\|^{2}+\left\|X_{0}\right\|^{2}\right) .
$$

By (3.7) and Lemma 2.1] $X_{t} \in \operatorname{Dom}\left(C^{1 / 2}\right)$ a.s. and $\mathbf{E}\left\|C^{1 / 2} X_{t}\right\|^{2}<+\infty$. Finally, using the inequality $\left\|C_{\varepsilon}^{1 / 2} X_{t}\right\| \leq\left\|C^{1 / 2} X_{t}\right\|$ and the property

$$
C_{\varepsilon}^{1 / 2} X_{t}=(I+\varepsilon C)^{-1} C^{1 / 2} X_{t} \rightarrow_{\varepsilon \rightarrow 0+} C^{1 / 2} X_{t},
$$

one concludes (3.1) from (3.7).

It follows from Itô's formula in a Hilbert space and Theorem 3.1 that

Corollary 3.2. Let $\operatorname{Dom}\left(C^{1 / 2}\right) \subset \operatorname{Dom}(G)$. Then there exists a unique strong topology solution of (1.1) which is weakly continuous in probability under the assumptions of Theorem 3.1.

Remark 3.3. A closer inspection of the proof of Theorem 3.1 reveals that the assertions of this theorem hold when $G_{n}=P_{n} G$ and for any $\varphi \in \operatorname{Dom}(G)$,

$$
2 \operatorname{Re}\langle\varphi, G \varphi\rangle+\sum_{k=1}^{m}\left\|L_{k} \varphi\right\|^{2} \leq 0 .
$$

Remark 3.4. Employing similar arguments to those used in the proof of Theorem 3.1, we can generalize the results given in Theorem 3.1 and Remark 3.3 to the case $m=+\infty$ under the hypothesis that for any $\phi \in \mathfrak{D}^{*}, \sum_{j=1}^{\infty}\left\|L_{j}^{*} \phi\right\|^{2}<+\infty$.

\section{RATE OF CONVERGENCE OF FINITE DIMENSIONAL APPROXIMATIONS}

This section deals with the last stage of the Galerkin method, i.e., with strong convergence results. More precisely, we derive the rate of convergence of $\mathbf{E}\left\langle X_{t, n}, A X_{t, n}\right\rangle$ to $\mathbf{E}\left\langle X_{t}, A X_{t}\right\rangle$, where $A$ belongs to a certain class of linear operators.

First, let us note that $A$ is an unbounded operator in many physics applications [19, 14]. Therefore we do not restrict ourselves here to the case $A \in B(\mathfrak{h})$. We also point out that the basic idea behind the proof of the main results of this part is the application of Theorem 3.1. Roughly speaking, we will combine the constraint given by the domain of $C^{\frac{1}{2}}$ with estimates introduced in Theorem 3.1

To be concrete, from now on we will suppose that

S1: There are an orthonormal basis of $\mathfrak{h}\left\{\varphi_{j}\right\}_{j \in \mathbf{Z}_{+}^{p}}, p \in \mathbb{N}$, and a positive real number $l_{C}$ such that the operator $C \varphi_{j}=|j|^{l_{C}} \varphi_{j}$ satisfies condition H7. 
In addition to the fact that assumption $\mathrm{S} 1$ frequently holds in applications in quantum optics (see, e.g., [6, 7] and Section 6), this case allows us to introduce a general procedure which can be useful in other situations. Notice that $p$ is interpreted as the number of modes or degree of freedom of physical systems.

For simplicity, we consider that $\mathfrak{h}_{n}$ is the linear span of $\left\{\varphi_{j}: \overrightarrow{0} \leq j \leq \vec{n}\right\}$. That is, we reduce ourselves to the Galerkin approximation with fixed basis. To state our next results precisely, we introduce the following notation.

Definition 4.1. Let $a$ and $b$ be nonnegative integer numbers. Assume that $l$ is a nonnegative real number. Then $O_{l, a, b}$ denotes the set of all linear operators $B: \operatorname{Dom}(B) \subset \mathfrak{h} \rightarrow \mathfrak{h}$ such that for any $k \in \mathbb{Z}_{+}^{p}, B \varphi_{k}$ belongs to the linear span of $\left\{\varphi_{j}\right\}_{j \in[k-a, k+b]}$ and $\left\|B \varphi_{k}\right\| \leq K \max \left\{|k|^{l}, 1\right\}$.

We are now ready to fulfill our objective of characterizing the convergence properties corresponding to Step 1 explained in Section 1.

Theorem 4.2. Let $A \in O_{l_{A}, n_{A}, m_{A}}, G \in O_{l_{G}, n_{G}, m_{G}}$ and $L_{j} \in O_{l_{L}, n_{L}, m_{L}}, j=$ $1, \ldots, m$. Suppose that $X_{0} \in \operatorname{Dom}\left(C^{1 / 2}\right) \subset \operatorname{Dom}(G)$ and $l_{C} \geq 2 \max \left\{l_{A}, l_{G}, 2 l_{L}\right\}$. Then under assumptions $S 1$ and $H 1$ to $H 6$, we have that for any $n>0$,

$$
\begin{aligned}
& \sup _{t \in[0, T]}\left|\mathbf{E}\left\langle X_{t}, A X_{t}\right\rangle-\mathbf{E}\left\langle X_{t, n}, A X_{t, n}\right\rangle\right| \leq K\left(\left\|C^{1 / 2} X_{0}\right\|^{2}+\left\|X_{0}\right\|^{2}\right) \\
& \quad *\left(\frac{1}{n^{l_{C}-l_{A}}}+\frac{\mathbf{1}_{n_{G}>0}}{n^{l_{C} / 2-l_{G}}}+\frac{\mathbf{1}_{n_{L}>0}+\mathbf{1}_{m_{L}>0}}{n^{l_{C} / 2-2 l_{L}}}+\frac{\mathbf{1}_{n_{G}>0}}{n^{l_{C}-2 l_{G}-l_{A}}}+\frac{\mathbf{1}_{n_{L}>0}+\mathbf{1}_{m_{L}>0}}{n^{l_{C}-4 l_{L}-l_{A}}}\right) .
\end{aligned}
$$

For expository purposes, before proving Theorem 4.2, we give Lemma 4.3.

Lemma 4.3. Let $\Phi \in O_{l, a, b}$. Then $\Phi^{*} \in O_{l, b, a}$. Furthermore, if $\bar{P}_{k}$ is the orthogonal projection of $\mathfrak{h}$ over the linear span of $\left\{\varphi_{j}\right\}_{j \in[\overrightarrow{0}, k]}$, then $\left\|\Phi \bar{P}_{k}\right\| \leq K|k|^{l}$ whenever $k \neq \overrightarrow{0}$.

Proof. Since $j \in[k-a, k+b]$ iff $k \in[j-b, j+a], \Phi^{*} \varphi_{k}$ belongs to the linear span of $\left\{\varphi_{j}\right\}_{j \in[k-b, k+a]}$. Let $k>0$. By the Cauchy-Schwarz inequality,

$$
\left\|\Phi \bar{P}_{k} z\right\|^{2} \leq K|k|^{2 l}\left(\sum_{i \in[\overrightarrow{0}, k]}\left|z^{i}\right|^{2}\right)^{\frac{1}{2}}\left(\sum_{i \in[\overrightarrow{0}, k]} \sum_{j \in[i-a-b, i+a+b] \cap[\overrightarrow{0}, k]}\left|z^{j}\right|^{2}\right)^{\frac{1}{2}},
$$

where $z \in \mathfrak{h}$. Thus $\left\|\Phi \bar{P}_{k} z\right\|^{2} \leq K|k|^{2 l}\|z\|^{2}$. Finally, if $S$ is the linear span of $\left\{\varphi_{j}\right\}_{j \in[0, k+a]}$, then

$$
\left\|\Phi^{*} \varphi_{k}\right\|=\sup _{\phi \in S, \phi \neq 0} \frac{\left\langle\Phi^{*} \varphi_{k}, \phi\right\rangle}{\|\phi\|} \leq\left\|\varphi_{k}\right\|\left\|\Phi \bar{P}_{k+a}\right\| .
$$

This implies that $\Phi^{*} \in O_{l, b, a}$.

Proof of Theorem 4.2. One easily obtains that we can reduce the study of the truncation error

$$
\left|\mathbf{E}\left\langle X_{t}, A X_{t}\right\rangle-\mathbf{E}\left\langle X_{t, n}, A X_{t, n}\right\rangle\right|
$$

to the analysis of the errors $H_{t}^{1}=\left|\mathbf{E}\left\langle X_{t}, A X_{t}\right\rangle-\mathbf{E}\left\langle P_{n} X_{t}, A P_{n} X_{t}\right\rangle\right|$ and $H_{t}^{2}=$ $\left|\mathbf{E}\left\langle P_{n} X_{t}, A P_{n} X_{t}\right\rangle-\mathbf{E}\left\langle X_{t, n}, A X_{t, n}\right\rangle\right|$. Then we will concentrate on estimating $H_{t}^{1}$ and $H_{t}^{2}$. 
Next, we will check that

$$
H_{t}^{1} \leq K n^{l_{A}-l_{C}}\left(\left\|C^{1 / 2} X_{0}\right\|^{2}+\left\|X_{0}\right\|^{2}\right) .
$$

To verify (4.1), we decompose $H_{t}^{1}$ as follows:

$$
\begin{aligned}
H_{t}^{1} \leq & \left|\mathbf{E}\left\langle P_{n} X_{t}, A\left(X_{t}-P_{n} X_{t}\right)\right\rangle\right|+\left|\mathbf{E}\left\langle X_{t}-P_{n} X_{t}, A P_{n} X_{t}\right\rangle\right| \\
& +\left|\mathbf{E}\left\langle X_{t}-P_{n} X_{t}, A\left(X_{t}-P_{n} X_{t}\right)\right\rangle\right| .
\end{aligned}
$$

Now, by $A \in O_{l_{A}, n_{A}, m_{A}}$,

$$
\begin{aligned}
\mid\left\langle X_{t}\right. & \left.-P_{n} X_{t}, A\left(X_{t}-P_{n} X_{t}\right)\right\rangle \mid \\
& \leq K \sum_{j \notin[\overrightarrow{0}, \vec{n}]}\left(\left|X_{t}^{j}\right||j|^{l_{A}} \sum_{k \in\left[j-n_{A}, j+m_{A}\right] \cap[\overrightarrow{0}, \vec{n}]^{c}}\left|X_{t}^{k}\right|\right) .
\end{aligned}
$$

Hence, using the Cauchy-Schwarz inequality and the fact that $k \in\left[j-n_{A}, j+m_{A}\right]$ iff $j \in\left[k-m_{A}, k+n_{A}\right]$, one concludes that

$$
\begin{aligned}
\mid\left\langle X_{t}\right. & \left.-P_{n} X_{t}, A\left(X_{t}-P_{n} X_{t}\right)\right\rangle \mid \\
& \leq K \sqrt{\sum_{j \notin[\overrightarrow{0}, \vec{n}]}\left|X_{t}^{j}\right|^{2}|j|^{l_{A}}} \sqrt{\sum_{j \notin[\overrightarrow{0}, \vec{n}]} \sum_{k \in\left[j-n_{A}, j+m_{A}\right] \cap[\overrightarrow{0}, \vec{n}]^{c}}\left|X_{t}^{k}\right|^{2}\left(|k|+p n_{A}\right)^{l_{A}}} \\
& \leq K \sum_{j \notin[\overrightarrow{0}, \vec{n}]}\left|X_{t}^{j}\right|^{2}|j|^{l_{A}} .
\end{aligned}
$$

Since $l_{C} \geq l_{A}$,

$$
\left|\left\langle X_{t}-P_{n} X_{t}, A\left(X_{t}-P_{n} X_{t}\right)\right\rangle\right| \leq K n^{l_{A}-l_{C}}\left\|C^{1 / 2} X_{t}\right\|^{2} .
$$

Repeating the arguments given above, we obtain

$$
\begin{aligned}
\mid\left\langle X_{t}\right. & \left.-P_{n} X_{t}, A P_{n} X_{t}\right\rangle \mid \\
& \leq \sum_{k \notin[\overrightarrow{0}, \vec{n}]} \sum_{j \in[\overrightarrow{0}, \vec{n}]}\left|X_{t}^{j}\right|\left|X_{t}^{k}\right|\left|\left\langle\varphi_{k}, A \varphi_{j}\right\rangle\right| \\
& \leq K \sqrt{\sum_{k \notin[\overrightarrow{0}, \vec{n}]}\left|X_{t}^{k}\right|^{2}} \sqrt{\sum_{j \in[0, \vec{n}],|j|>n-m_{A} p}\left|X_{t}^{j}\right|^{2} \max \left\{|j|^{2 l_{A}}, 1\right\} .}
\end{aligned}
$$

It follows from $l_{C} \geq 2 l_{A}$ that for every $n>m_{A} p$,

$$
\left|\left\langle X_{t}-P_{n} X_{t}, A P_{n} X_{t}\right\rangle\right| \leq K n^{l_{A}-l_{C}}\left\|C^{1 / 2} X_{t}\right\|^{2} .
$$

Furthermore, (4.4) implies that for any $n \leq m_{A} p$,

$$
\begin{aligned}
\left|\left\langle X_{t}-P_{n} X_{t}, A P_{n} X_{t}\right\rangle\right| & \leq K n^{l_{A}-l_{C} / 2}\left\|C^{1 / 2} X_{t}\right\|\left\|X_{t}\right\| \\
& \leq K n^{l_{A}-l_{C}}\left\|C^{1 / 2} X_{t}\right\|\left\|X_{t}\right\| .
\end{aligned}
$$

Finally, since $\left\langle P_{n} X_{t}, A\left(X_{t}-P_{n} X_{t}\right)\right\rangle=\left\langle A^{*} P_{n} X_{t}, X_{t}-P_{n} X_{t}\right\rangle$, combining Lemma 4.3, inequalities (4.5), (4.6), (4.3) and Theorem [3.1, we arrive at (4.1). 
Now, we go on estimating $H_{t}^{2}$. To treat this term, we first note that

$$
\begin{aligned}
H_{t}^{2} \leq & \left(\left(\mathbf{E}\left\|A^{*} X_{t, n}\right\|^{2}\right)^{\frac{1}{2}}+\left(\mathbf{E}\left\|A X_{t, n}\right\|^{2}\right)^{\frac{1}{2}}\right)\left(\mathbf{E}\left\|P_{n} X_{t}-X_{t, n}\right\|^{2}\right)^{\frac{1}{2}} \\
& +\left\|A P_{n}\right\| \mathbf{E}\left\|P_{n} X_{t}-X_{t, n}\right\|^{2}
\end{aligned}
$$

Since

$$
\begin{aligned}
\left\|A^{*} X_{t, n}\right\|^{2} \leq & K\left(\sum_{j \in[\overrightarrow{0}, \vec{n}]}\left|X_{t, n}^{j}\right|^{2} \max \left\{|j|^{l_{A}}, 1\right\}^{2}\right)^{\frac{1}{2}} \\
& *\left(\sum_{j \in[\overrightarrow{0}, \vec{n}]} \sum_{k \in\left[j-n_{A}-m_{A}, j+n_{A}+m_{A}\right] \cap[\overrightarrow{0}, \vec{n}]}\left|X_{t, n}^{k}\right|^{2} \max \left\{|k|^{l_{A}}, 1\right\}^{2}\right)^{\frac{1}{2}},
\end{aligned}
$$

$$
\left\|A^{*} X_{t, n}\right\|^{2} \leq K \sum_{j \in[\overrightarrow{0}, \vec{n}]}\left|X_{t, n}^{j}\right|^{2} \max \left\{|j|^{l_{A}}, 1\right\}^{2} \leq K\left(\left\|C^{\frac{1}{2}} X_{t, n}\right\|^{2}+\left|X_{t, n}^{\overrightarrow{0}}\right|^{2}\right) .
$$

Therefore, Lemma 4.3 leads to

$$
\left\|A X_{t, n}\right\|^{2} \leq K\left(\left\|C^{\frac{1}{2}} X_{t, n}\right\|^{2}+\left|X_{t, n}^{\overrightarrow{0}}\right|^{2}\right) .
$$

Using Theorem 3.1 Lemma 4.3 (4.8) and (4.9) in 4.7), it follows that

$$
\begin{aligned}
H_{t}^{2} \leq & K \sqrt{\left\|C^{\frac{1}{2}} P_{n} X_{0}\right\|^{2}+\left\|X_{0}\right\|^{2}} \sqrt{\mathbf{E}\left\|P_{n} X_{t}-X_{t, n}\right\|^{2}} \\
& +K n^{l_{A}} \mathbf{E}\left\|P_{n} X_{t}-X_{t, n}\right\|^{2} .
\end{aligned}
$$

Consequently, it remains to bound $h(t)=\mathbf{E}\left\|P_{n} X_{t}-X_{t, n}\right\|^{2}$. Applying Corollary 3.2, one concludes that

$$
P_{n} X_{t}-X_{t, n}=\int_{0}^{t}\left(P_{n} G X_{s}-G_{n} X_{s, n}\right) d s+\sum_{k=1}^{m} \int_{0}^{t} L_{k, n}\left(X_{s}-X_{s, n}\right) d W_{s}^{k} .
$$

Therefore, Itô's formula yields

$$
\begin{aligned}
h(t)= & 2 \mathbf{E} \int_{0}^{t} \operatorname{Re}\left\langle P_{n} X_{s}-X_{s, n}, P_{n} G X_{s}-G_{n} X_{s, n}\right\rangle d s \\
& +\sum_{k=1}^{m} \mathbf{E} \int_{0}^{t}\left\|L_{k, n}\left(X_{s}-X_{s, n}\right)\right\|^{2} d s .
\end{aligned}
$$

Thus, we deduce from (1.6) that $\mathbf{E}\left\|P_{n} X_{t}-X_{t, n}\right\|^{2}=\sum_{k=1}^{4} J_{t}^{k}$, where

$$
\begin{gathered}
J_{t}^{1}=2 \mathbf{E} \int_{0}^{t} \operatorname{Re}\left\langle P_{n} X_{s}-X_{s, n},\left(P_{n} G-G_{n}\right) P_{n} X_{s}\right\rangle d s \\
J_{t}^{2}=2 \mathbf{E} \int_{0}^{t} \operatorname{Re}\left\langle P_{n} X_{s}-X_{s, n}, P_{n} G\left(X_{s}-P_{n} X_{s}\right)\right\rangle d s \\
J_{t}^{3}=\sum_{k=1}^{m} \mathbf{E} \int_{0}^{t}\left\|L_{k, n}\left(X_{s}-P_{n} X_{s}\right)\right\|^{2} d s
\end{gathered}
$$


and $J_{t}^{4}=2 \sum_{k=1}^{m} 2 \mathbf{E} \int_{0}^{t} \operatorname{Re}\left\langle L_{k, n}\left(X_{s}-P_{n} X_{s}\right), L_{k, n}\left(P_{n} X_{s}-X_{s, n}\right)\right\rangle d s$.

Fix $m_{L}=0$. Then $J_{t}^{1}=0$, because $\left(P_{n} G-G_{n}\right) P_{n} X_{s}=0$. In the other case, i.e., $m_{L} \geq 1$, combining Lemma 4.3 and Theorem 3.1 we obtain

$$
\begin{aligned}
& \mathbf{E}\left\|\left(P_{n} G-G_{n}\right) P_{n} X_{s}\right\|^{2} \\
& \quad \leq \frac{m}{4} \sum_{k=1}^{m} \mathbf{E}\left\|\left(P_{n} L_{k}^{*} P_{n} L_{k}-P_{n} L_{k}^{*} L_{k}\right)\left(P_{n} X_{s}-P_{n-m_{L}} X_{s}\right)\right\|^{2} \\
& \quad \leq K n^{4 l_{L}} \mathbf{E}\left\|P_{n} X_{s}-P_{n-m_{L}} X_{s}\right\|^{2} \\
& \quad \leq K n^{4 l_{L}-l_{C}}\left(\left\|C^{1 / 2} X_{0}\right\|^{2}+\left\|X_{0}\right\|^{2}\right),
\end{aligned}
$$

which yields

$$
J_{t}^{1} \leq K \mathbf{1}_{m_{L}>0} n^{2 l_{L}-\frac{l_{C}}{2}}\left(\left\|C^{\frac{1}{2}} X_{0}\right\|^{2}+\left\|X_{0}\right\|^{2}\right)^{\frac{1}{2}} \int_{0}^{t} \mathbf{E}^{\frac{1}{2}}\left(\left\|P_{n} X_{s}-X_{s, n}\right\|^{2}\right) d s .
$$

It is worth mentioning that in previous inequalities if $n-m_{L}<0$, then we put $P_{n-m_{L}}=0$.

Clearly, if $n_{G}=0$, resp. $n_{L}=0$, then $J_{t}^{2}=0$, resp. $J_{t}^{3}=0$. Moreover, from (4.3), Lemma 4.3,

$$
\left\|P_{n} G\left(X_{s}-P_{n} X_{s}\right)\right\|^{2}=\left\langle X_{s}-P_{n} X_{s}, G^{*} P_{n} G\left(X_{s}-P_{n} X_{s}\right)\right\rangle
$$

and $\left\|L_{k, n}\left(X_{s}-P_{n} X_{s}\right)\right\|^{2}=\left\langle X_{s}-P_{n} X_{s}, L_{k}^{*} P_{n} L_{k}\left(X_{s}-P_{n} X_{s}\right)\right\rangle$, it follows that

$$
\begin{aligned}
J_{t}^{2}+J_{t}^{3} \leq & \mathbf{1}_{n_{L}>0} K n^{2 l_{L}-l_{C}}\left(\left\|C^{\frac{1}{2}} X_{0}\right\|^{2}+\left\|X_{0}\right\|^{2}\right) \\
& +\mathbf{1}_{n_{G}>0} K n^{l_{G}-l_{C} / 2}\left(\left\|C^{\frac{1}{2}} X_{0}\right\|^{2}+\left\|X_{0}\right\|^{2}\right)^{\frac{1}{2}} \int_{0}^{t} \mathbf{E}^{\frac{1}{2}}\left(\left\|P_{n} X_{s}-X_{s, n}\right\|^{2}\right) d s .
\end{aligned}
$$

Since

$$
\begin{aligned}
& \left\langle L_{k, n}\left(X_{s}-P_{n} X_{s}\right), L_{k, n}\left(P_{n} X_{s}-X_{s, n}\right)\right\rangle \\
& \quad=\left\langle L_{k}^{*} P_{n} L_{k}\left(X_{s}-P_{n} X_{s}\right), P_{n} X_{s}-X_{s, n}\right\rangle,
\end{aligned}
$$

we also have

$$
J_{t}^{4} \leq \mathbf{1}_{n_{L}>0} K n^{2 l_{L}-l_{C} / 2}\left(\left\|C^{\frac{1}{2}} X_{0}\right\|^{2}+\left\|X_{0}\right\|^{2}\right)^{\frac{1}{2}} \int_{0}^{t} \mathbf{E}^{\frac{1}{2}}\left(\left\|P_{n} X_{s}-X_{s, n}\right\|^{2}\right) d s .
$$

To summarize, we have obtained that

$$
\begin{aligned}
h(t) \leq & K \frac{\mathbf{1}_{n_{L}>0}}{n^{l_{C}-2 l_{L}}}\left(\left\|C^{\frac{1}{2}} X_{0}\right\|^{2}+\left\|X_{0}\right\|^{2}\right) \\
& +K\left(\left\|C^{\frac{1}{2}} X_{0}\right\|^{2}+\left\|X_{0}\right\|^{2}\right)^{\frac{1}{2}} *\left(\frac{\mathbf{1}_{n_{L}>0}+\mathbf{1}_{m_{L}>0}}{n^{l_{C} / 2-2 l_{L}}}+\frac{\mathbf{1}_{n_{G}>0}}{n^{l_{C} / 2-l_{G}}}\right) \int_{0}^{t} \sqrt{h(s)} d s .
\end{aligned}
$$

Therefore, according to Lemma 2.2

$$
h(t) \leq K\left(\left\|C^{\frac{1}{2}} X_{0}\right\|^{2}+\left\|X_{0}\right\|^{2}\right)\left(\frac{\mathbf{1}_{n_{L}>0}+\mathbf{1}_{m_{L}>0}}{n^{l_{C} / 2-2 l_{L}}}+\frac{\mathbf{1}_{n_{G}>0}}{n^{l_{C} / 2-l_{G}}}\right)^{2} .
$$

Then the required result follows from (4.10).

To handle the case $A \in B(\mathfrak{h}) \backslash \bigcup_{n_{A}, m_{A}=0}^{\infty} O_{0, n_{A}, m_{A}}$, we present the following theorem. 
Theorem 4.4. Let $A \in A \in B(\mathfrak{h}), G \in O_{l_{G}, n_{G}, m_{G}}$ and $L_{j} \in O_{l_{L}, n_{L}, m_{L}}, j=$ $1, \ldots, m$. Suppose that $X_{0} \in \operatorname{Dom}\left(C^{1 / 2}\right) \subset \operatorname{Dom}(G)$ and $l_{C} \geq 2 \max \left\{l_{G}, 2 l_{L}\right\}$. Then under assumptions $S 1$ and H1-H6, we have that for any $n>0$,

$$
\begin{aligned}
& \sup _{t \in[0, T]}\left|\mathbf{E}\left\langle X_{t}, A X_{t}\right\rangle-\mathbf{E}\left\langle X_{t, n}, A X_{t, n}\right\rangle\right| \\
& \quad \leq K\left(\left\|C^{1 / 2} X_{0}\right\|^{2}+\left\|X_{0}\right\|^{2}\right)\left(\frac{1}{n^{l_{C} / 2}}+\frac{\mathbf{1}_{n_{G}>0}}{n^{l_{C} / 2-l_{G}}}+\frac{\mathbf{1}_{n_{L}>0}+\mathbf{1}_{m_{L}>0}}{n^{l_{C} / 2-2 l_{L}}}\right) .
\end{aligned}
$$

Proof. Let $H_{t}^{1}$ and $H_{t}^{2}$ be defined as in the proof of Theorem 4.2 Hence

$$
H_{t}^{1} \leq\|A\| \mathbf{E}\left(2\left\|P_{n} X_{t}\right\|\left\|X_{t}-P_{n} X_{t}\right\|+\left\|X_{t}-P_{n} X_{t}\right\|^{2}\right)
$$

and

$$
\begin{aligned}
H_{t}^{2} \leq & \|A\|\left(\left(\mathbf{E}\left\|X_{t, n}\right\|^{2}\right)^{\frac{1}{2}}+\left(\mathbf{E}\left\|X_{t, n}\right\|^{2}\right)^{\frac{1}{2}}\right)\left(\mathbf{E}\left\|P_{n} X_{t}-X_{t, n}\right\|^{2}\right)^{\frac{1}{2}} \\
& +\|A\| \mathbf{E}\left\|P_{n} X_{t}-X_{t, n}\right\|^{2} .
\end{aligned}
$$

Therefore, applying (4.3) and (4.11), the theorem follows.

\section{Rate of convergence of the explicit Euler scheme}

In this section our aim is to progress in the theoretical understanding of Step 2 of the procedure given in Section 1. Indeed, we will address the numerical simulation of $\mathbf{E}\left\langle X_{t, n}, A X_{t, n}\right\rangle$ using the explicit Euler scheme, i.e., the scheme $Z_{\cdot, n}$ recalled in Section 1.

Because of its instability, the explicit Euler scheme $Z$ does not present a good performance when (1.5) is a stiff SDE [16, 13]. Though this situation often appears in quantum mechanics, the error analysis presented here can be a useful pattern for studying more complex numerical schemes, such as implicit Euler methods [16. 13 26], Euler-Exponential methods [17 and those resulting of the application of the above schemes to the solution of the SDE satisfied by $X_{t, n} /\left\|X_{t, n}\right\|$.

We start the section with Theorem 5.1 which provides the rate of convergence of the explicit Euler scheme. Next, we focus on a numerical method based on the extrapolation of this scheme.

Theorem 5.1. Suppose that $\mu$ is a positive constant and conditions of Theorem 4.2 hold. Let $\left(c_{n}\right)_{n}$ be a sequence of real numbers such that

S2: for any $n \geq 1$ and $x \in \mathfrak{h}_{n},\left\|G_{n} P_{n}\right\|^{2} \leq c_{n}$ and

$$
\left\|C^{1 / 2} G_{n} x\right\|^{2} \leq K c_{n}\left(\left\|C^{1 / 2} x\right\|^{2}+\|x\|^{2}\right) .
$$

Moreover, assume that

S3: the operator given by $C_{1} \varphi_{n}=|n|^{l_{C_{1}}} \varphi_{n}$, where $l_{C_{1}}$ is a positive real number such that $l_{C} \geq l_{C_{1}}+4 \max \left\{l_{G}, l_{L}\right\}$ and $l_{C_{1}} \geq l_{A}$, fulfills condition $\mathrm{H}^{7}$. 


$$
\begin{aligned}
& \text { If } c_{n} \leq \mu M \text {, then for any } j=0, \ldots, M, \\
& \begin{aligned}
\left|\mathbf{E}\left\langle X_{T_{j}}, A X_{T_{j}}\right\rangle-\mathbf{E}\left\langle Z_{T_{j}, n}^{M}, A Z_{T_{j}, n}^{M}\right\rangle\right| \\
\leq K\left(\left\|C^{1 / 2} X_{0}\right\|^{2}+\left\|X_{0}\right\|^{2}\right) \\
\quad *\left(\frac{T}{M}+\frac{\mathbf{1}_{n_{G}>0}}{n^{l_{C} / 2-l_{G}}}\right. \\
\left.\quad+\frac{\mathbf{1}_{n_{L}>0}+\mathbf{1}_{m_{L}>0}}{n^{l_{C} / 2-2 l_{L}}}+\frac{\mathbf{1}_{n_{G}>0}}{n^{l_{C}-2 l_{G}-l_{A}}}+\frac{\mathbf{1}_{n_{L}>0}+\mathbf{1}_{m_{L}>0}}{n^{l_{C}-4 l_{L}-l_{A}}}+\frac{1}{n^{l_{C}-l_{A}}}\right) .
\end{aligned}
\end{aligned}
$$

As we will illustrate in Section 6, the hypotheses of Theorem 5.1 are general enough for applications in quantum optics. The underlying idea in the proof of Theorem 5.1 is to refine standard arguments employed to prove the rate of convergence of classical weak numerical schemes for finite dimensional SDEs [23, 24, 16, 13. keeping in mind the characteristics of our case, e.g., Remark 5.2 and Lemma 5.3.

Remark 5.2. One specific feature which is used in the proof is that for each $x \in \mathfrak{h}_{n}$, $t \in[0, T]$ and $s \in[0, t]$,

$$
\left\langle x, \Phi_{t, n}^{s} x\right\rangle=\mathbf{E}\left\langle Y_{t, n}^{s, x}, A Y_{t, n}^{s, x}\right\rangle,
$$

where for every $t \in[s, T]$

$$
Y_{t, n}^{s, x}=x+\int_{s}^{t} G_{n} Y_{u, n}^{s, x} d u+\sum_{k=1}^{m} \int_{s}^{t} L_{k, n} Y_{u, n}^{s, x} d W_{u}^{k}
$$

and

$$
\Phi_{t, n}^{s}=P_{n} A P_{n}+\int_{s}^{t} \mathcal{L}_{n}\left(\Phi_{u, n}^{s}\right) d u
$$

with $\mathcal{L}_{n}: B\left(\mathfrak{h}_{n}\right) \rightarrow B\left(\mathfrak{h}_{n}\right)$ given by

$$
\mathcal{L}_{n}(X)=P_{n} G_{n}^{*} X+X G_{n} P_{n}+\sum_{k=1}^{m} P_{n} L_{k}^{*} P_{n} X P_{n} L_{k} P_{n} .
$$

For the sake of simplicity, we use the shorthand notation $\Phi_{t-s, n}$ to mean $\Phi_{t-s, n}^{0}$.

Lemma 5.3. Suppose that the hypotheses of Theorem 5.1 hold. Consider $B_{1}, B_{2} \in$ $O_{l_{B}, n_{B}, m_{B}}$ satisfying $l_{C} \geq l_{C_{1}}+2 l_{B}$. Then

$$
\left|\mathbf{E}\left\langle B_{1} Z_{T_{j}, n}, \Phi_{T_{\kappa}-s, n} B_{2} Z_{T_{j}, n}\right\rangle\right| \leq K\left(\left\|C^{\frac{1}{2}} X_{0}\right\|^{2}+\left\|X_{0}\right\|^{2}\right)
$$

whenever $T_{j} \leq s \leq T_{\kappa}$.

Proof. First, we establish estimates on $Z_{t, n}$. Next, using them, we obtain (5.4).

By Itô's formula, we have that for every $t \in] T_{j}, T_{j+1}$ ]

$$
\begin{aligned}
& \mathbf{E}\left\|Z_{t, n}\right\|^{2} \\
& =\mathbf{E}\left\|Z_{T_{j}, n}\right\|^{2}+\mathbf{E} \int_{T_{j}}^{t} 2 \operatorname{Re}\left\langle Z_{s, n}, G_{n} Z_{T_{j}, n}\right\rangle d s+\sum_{k=1}^{m} \mathbf{E} \int_{T_{j}}^{t}\left\|L_{k, n} Z_{T_{j}, n}\right\|^{2} d s \\
& =\mathbf{E}\left\|Z_{T_{j}, n}\right\|^{2}+\mathbf{E} \int_{T_{j}}^{t} 2 \operatorname{Re}\left\langle G_{n} Z_{T_{j}, n}\left(s-T_{j}\right), G_{n} Z_{T_{j}, n}\right\rangle d s .
\end{aligned}
$$


Hence $\mathbf{E}\left\|Z_{t, n}\right\|^{2} \leq \mathbf{E}\left\|Z_{T_{j}, n}\right\|^{2}\left(1+\left\|G_{n} P_{n}\right\|^{2} \frac{\left(t-T_{j}\right)^{2}}{2}\right)$. Therefore

$$
\mathbf{E}\left\|Z_{t, n}\right\|^{2} \leq\left\|Z_{0, n}\right\|^{2}\left(1+c_{n} \frac{T^{2}}{2 M^{2}}\right)^{j} \leq\left\|Z_{0, n}\right\|^{2} e^{\frac{c_{n} T^{2}}{2 M}} \leq\left\|Z_{0, n}\right\|^{2} e^{\frac{\mu}{2} T^{2}} .
$$

On the other hand, for every $\left.t \in] T_{j}, T_{j+1}\right]$

$$
C^{\frac{1}{2}} Z_{t, n}=C^{\frac{1}{2}} Z_{T_{j}, n}+\int_{T_{j}}^{t} C^{\frac{1}{2}} G_{n} Z_{T_{j}, n} d s+\sum_{k=1}^{m} \int_{T_{j}}^{t} C^{\frac{1}{2}} L_{k, n} Z_{T_{j}, n} d W_{s}^{k}
$$

Therefore, from Itô's formula it follows that

$$
\begin{aligned}
\mathbf{E}\left\|C^{\frac{1}{2}} Z_{t, n}\right\|^{2}= & \mathbf{E}\left\|C^{\frac{1}{2}} Z_{T_{j}, n}\right\|^{2}+\mathbf{E} \int_{T_{j}}^{t} 2 \operatorname{Re}\left\langle C^{\frac{1}{2}} Z_{s, n}, C^{\frac{1}{2}} G_{n} Z_{T_{j}, n}\right\rangle d s \\
& +\sum_{k=1}^{m} \mathbf{E} \int_{T_{j}}^{t}\left\|C^{\frac{1}{2}} L_{k, n} Z_{T_{j}, n}\right\|^{2} d s .
\end{aligned}
$$

Thus (5.5) and hypothesis S1 yield

$$
\begin{aligned}
\mathbf{E}\left\|C^{\frac{1}{2}} Z_{t, n}\right\|^{2} \leq & \left(1+\frac{K T}{M}\right) \mathbf{E}\left\|C^{\frac{1}{2}} Z_{T_{j}, n}\right\|^{2}+\frac{K T}{M}\left\|Z_{0, n}\right\|^{2} \\
& +\mathbf{E} \int_{T_{j}}^{t} 2 \operatorname{Re}\left\langle C^{\frac{1}{2}}\left(Z_{s, n}-Z_{T_{j}, n}\right), C^{\frac{1}{2}} G_{n} Z_{T_{j}, n}\right\rangle d s .
\end{aligned}
$$

Hence combining S2, the linearity of (1.5) and the fact that $c_{n} \leq \mu M$, we obtain

$$
\mathbf{E}\left\|C^{\frac{1}{2}} Z_{t, n}\right\|^{2} \leq\left(1+\frac{K T}{M}\right) \mathbf{E}\left\|C^{\frac{1}{2}} Z_{T_{j}, n}\right\|^{2}+\frac{K T}{M}\left\|Z_{0, n}\right\|^{2} .
$$

Then for any $\left.t \in] T_{j}, T_{j+1}\right]$,

$$
\begin{aligned}
\mathbf{E}\left\|C^{\frac{1}{2}} Z_{t, n}\right\|^{2} & \leq\left(1+\frac{K T}{M}\right)^{j} \mathbf{E}\left\|C^{\frac{1}{2}} Z_{0, n}\right\|^{2}+\sum_{k=0}^{j-1}\left(1+\frac{K T}{M}\right)^{k} \frac{K T}{M}\left\|Z_{0, n}\right\|^{2} \\
& \leq \mathbf{E}\left\|C^{\frac{1}{2}} Z_{0, n}\right\|^{2} e^{K}+K\left\|Z_{0, n}\right\|^{2}\left(\left(1+\frac{K}{M}\right)^{M}-1\right) \\
& \leq K \mathbf{E}\left\|C^{\frac{1}{2}} Z_{0, n}\right\|^{2}+K\left\|Z_{0, n}\right\|^{2}\left(e^{K}-1\right) .
\end{aligned}
$$

In the remainder of the proof we verify (5.4). Using the polarization identity, (5.1) and the property $\Phi_{t-s, n}=\Phi_{t, n}^{s}$, we obtain

$$
\begin{aligned}
4 \mid \mathbf{E} & \left\langle B_{1} Z_{T_{j}, n}, \Phi_{T_{\kappa}-s, n} B_{2} Z_{T_{j}, n}\right\rangle \mid \\
& =\left|\sum_{k=0}^{3} i^{-k} \mathbf{E}\left\langle\left(B_{1}+i^{k} B_{2}\right) Z_{T_{j}, n}, \Phi_{T_{\kappa}-s, n}\left(B_{1}+i^{k} B_{2}\right) Z_{T_{j}, n}\right\rangle\right| \\
& \leq \sum_{k=0}^{3}\left|\mathbf{E}\left\langle\widetilde{Y}_{n, k}, A \widetilde{Y}_{n, k}\right\rangle\right|,
\end{aligned}
$$


with $\widetilde{Y}_{n, k}=Y_{T_{\kappa}, n}^{s,\left(B_{1}+i^{k} B_{2}\right) Z_{T_{j}, n}}$. Proceeding as in the proof of Theorem 4.2 one arrives at

$$
\begin{aligned}
& \left|\mathbf{E}\left(\left\langle\widetilde{Y}_{n, k}, A \widetilde{Y}_{n, k}\right\rangle / F_{s}\right)\right| \\
& \quad \leq K \mathbf{E}\left(\sum_{l \in[\overrightarrow{0}, \vec{n}]}\left|\widetilde{Y}_{n, k}^{l}\right|^{2} \max \left\{|l|^{l_{A}}, 1\right\} / F_{T_{s}}\right) \\
& \quad \leq K \mathbf{E}\left(\left\|C_{1}^{\frac{1}{2}} \widetilde{Y}_{n, k}\right\|^{2}+\left\|\tilde{Y}_{n, k}^{\overrightarrow{0}}\right\|^{2} / F_{T_{s}}\right) .
\end{aligned}
$$

It follows from Theorem 3.1 and the Markov property of $Y$ that

$$
\left|\mathbf{E}\left(\left\langle\widetilde{Y}_{n, k}, A \widetilde{Y}_{n, k}\right\rangle / F_{s}\right)\right| \leq K\left(\left\|C_{1}^{\frac{1}{2}}\left(B_{1}+i^{k} B_{2}\right) Z_{T_{j}, n}\right\|^{2}+\left\|\left(B_{1}+i^{k} B_{2}\right) Z_{T_{j}, n}\right\|^{2}\right) .
$$

Since $l_{C} \geq l_{C_{1}}+2 l_{B}$,

$$
\left|\mathbf{E}\left(\left\langle\widetilde{Y}_{n, k}, A \widetilde{Y}_{n, k}\right\rangle / F_{s}\right)\right| \leq K\left(\left\|C^{\frac{1}{2}} Z_{T_{j}, n}\right\|^{2}+\left\|Z_{T_{j}, n}\right\|^{2}\right) .
$$

Then we conclude the proof of (5.4) by an application of the estimates obtained in the first part.

Proof of Theorem 5.1. The error $\left|\mathbf{E}\left\langle X_{T_{j}}, A X_{T_{j}}\right\rangle-\mathbf{E}\left\langle Z_{T_{j}, n}^{M}, A Z_{T_{j}, n}^{M}\right\rangle\right|$ can be decomposed into the sum of the error $\left|\mathbf{E}\left\langle X_{T_{j}}, A X_{T_{j}}\right\rangle-\mathbf{E}\left\langle X_{T_{j}, n}, A X_{T_{j}, n}\right\rangle\right|$, which arises in Step 1, and the discretization error

$$
\left|\mathbf{E}\left\langle X_{T_{j}, n}, A X_{T_{j}, n}\right\rangle-\mathbf{E}\left\langle Z_{T_{j}, n}^{M}, A Z_{T_{j}, n}^{M}\right\rangle\right| .
$$

Theorem 4.2 provides estimates for the first term, so that it remains to bound the discretization error.

To this end, we introduce the functions $u_{n}:\left[0, T_{j}\right] \times \mathfrak{h}_{n} \times \mathfrak{h}_{n} \rightarrow \mathbb{C}, n \in \mathbb{N}$, defined by

$$
u_{n}(s, x, y)=\left\langle\bar{x}, \Phi_{T_{j}, n}^{s} y\right\rangle
$$

where $\bar{x}=\sum_{j \in[\overrightarrow{0}, \vec{n}]} \overline{x_{j}} \varphi_{j}$ whenever $x=\sum_{j \in[\overrightarrow{0}, \vec{n}]} x_{j} \varphi_{j}$. Recall that $\left\{\varphi_{j}: \overrightarrow{0} \leq j \leq \vec{n}\right\}$ is an orthogonal basis of $\mathfrak{h}_{n}$. It follows from (5.1) that

$$
\begin{aligned}
& \mathbf{E}\left\langle Z_{T_{j}, n}, A Z_{T_{j}, n}\right\rangle-\mathbf{E}\left\langle X_{T_{j}, n}, A X_{T_{j}, n}\right\rangle \\
& =\mathbf{E}\left(u_{n}\left(T_{j}, \overline{Z_{T_{j}, n}}, Z_{T_{j}, n}\right)-u_{n}\left(0, \overline{X_{0, n}}, X_{0, n}\right)\right) \\
& =\sum_{k=0}^{j-1} \delta_{k},
\end{aligned}
$$

where $\delta_{k}=\mathbf{E}\left(u_{n}\left(T_{k+1}, \overline{Z_{T_{k+1}, n}}, Z_{T_{k+1}, n}\right)-u_{n}\left(T_{k}, \overline{Z_{T_{k}, n}}, Z_{T_{k}, n}\right)\right)$. 
Now, Itô's formula and (5.3) yield

$$
\begin{aligned}
\delta_{k}= & \mathbf{E} \int_{T_{k}}^{T_{k+1}}\left\langle G_{n}\left(Z_{T_{k}, n}-Z_{s, n}\right), \Phi_{T_{j}-s, n} Z_{s, n}\right\rangle d s \\
& +\mathbf{E} \int_{T_{k}}^{T_{k+1}}\left\langle Z_{s, n}, \Phi_{T_{j}-s, n} G_{n}\left(Z_{T_{k}, n}-Z_{s, n}\right)\right\rangle d s \\
& +\sum_{l=1}^{m} \mathbf{E} \int_{T_{k}}^{T_{k+1}}\left\langle L_{l, n} Z_{T_{k}, n}, \Phi_{T_{j}-s, n} L_{l, n} Z_{T_{k}, n}\right\rangle d s \\
& -\sum_{l=1}^{m} \mathbf{E} \int_{T_{k}}^{T_{k+1}}\left\langle L_{l, n} Z_{s, n}, \Phi_{T_{j}-s, n} L_{l, n} Z_{s, n}\right\rangle d s .
\end{aligned}
$$

Therefore, a simple calculation leads to

$$
\delta_{k}=-\sum_{\left(B_{1}, B_{2}, r\right) \in \mathcal{S}} \int_{T_{k}}^{T_{k+1}} \mathbf{E}\left\langle B_{1} Z_{T_{k}, n}, \Phi_{T_{j}-s, n} B_{2} Z_{T_{k}, n}\right\rangle\left(s-T_{k}\right)^{r} d s,
$$

where $\mathcal{S}$ is the set formed by the following triplets: $\left(G_{n}^{2}, I, 1\right),\left(I, G_{n}^{2}, 1\right),\left(G_{n}^{2}, G_{n}, 2\right)$, $\left(G_{n}, G_{n}^{2}, 2\right),\left(G_{n} L_{l, n}, L_{l, n}, 1\right),\left(L_{l, n}, G_{n} L_{l, n}, 1\right),\left(L_{l, n}, L_{l, n} G_{n}, 1\right),\left(L_{l, n} G_{n}, L_{l, n}, 1\right)$, $\left(L_{l, n} G_{n}, L_{l, n} G_{n}, 2\right)$ and $\left(L_{l, n} L_{l_{1}, n}, L_{l, n} L_{l_{1}, n}, 1\right)$, with $l, l_{1}=1, \ldots, m$. Thus Lemma 5.3 completes the proof.

To improve the weak convergence order of the Euler scheme, Talay and Tubaro generalized in [25] the Romberg extrapolation methods to the context of stochastic differential equations. Inspired by this work, we next develop a second-order scheme.

Theorem 5.4. Suppose that in addition to the hypotheses of Theorem 5.1 we have

S4: the operator given by $C_{2} \varphi_{n}=|n|^{l_{C_{2}}} \varphi_{n}$, where $l_{C_{2}}$ is a positive real number such that $l_{C_{1}} \geq l_{C_{2}}+4 \max \left\{l_{G}, l_{L}\right\}$ and $l_{C_{2}} \geq l_{A}$, fulfills condition $H^{7}$.

Then for any $j=0, \ldots, M$,

$$
\begin{array}{r}
\left|\mathbf{E}\left\langle X_{T_{j}^{M}}, A X_{T_{j}^{M}}\right\rangle-2 \mathbf{E}\left\langle Z_{T_{2 j}^{2 M}, n}^{2 M}, A Z_{T_{2 j}^{2 M}, n}^{2 M}\right\rangle+\mathbf{E}\left\langle Z_{T_{j}^{M}, n}^{M}, A Z_{T_{j}^{M}, n}^{M}\right\rangle\right| \\
\leq K\left(\left\|C^{1 / 2} X_{0}\right\|^{2}+\left\|X_{0}\right\|^{2}\right)\left(\frac{T^{2}}{M^{2}}+\frac{\mathbf{1}_{n_{G}>0}}{n^{l_{C} / 2-l_{G}}}+\frac{\mathbf{1}_{n_{L}>0+\mathbf{1}_{m_{L}>0}}}{n^{l_{C} / 2-2 l_{L}}}\right. \\
\left.+\frac{\mathbf{1}_{n_{G}>0}}{n^{l_{C}-2 l_{G}-l_{A}}}+\frac{\mathbf{1}_{n_{L}>0}+\mathbf{1}_{m_{L}>0}}{n^{l_{C}-4 l_{L}-l_{A}}}+\frac{1}{n^{l_{C}-l_{A}}}\right)
\end{array}
$$

provided that $c_{n} \leq \mu M$.

For expository purposes, before proving Theorem 5.4 we give the following lemma.

Lemma 5.5. Suppose that the hypotheses of Theorem 5.4 hold. Assume that $B \in$ $O_{l_{B}, n_{B}, m_{B}}$ and that $B_{k} \in O_{l_{B_{1}}, n_{B_{1}}, m_{B_{1}}}$ for $k=1, \ldots, 4$. If $l_{C} \geq l_{C_{1}}+2 l_{B_{1}}$ and $l_{C_{1}} \geq l_{C_{2}}+2 l_{B}$, then

$$
\left|\mathbf{E}\left\langle B Y_{T_{j}, n}^{s, B_{1} Z_{s, n}+B_{2} Z_{T_{j}, n}}, \Phi_{T_{\kappa}-\bar{s}, n} B Y_{T_{j}, n}^{s, B_{3} Z_{s, n}+B_{4} Z_{T_{j}, n}}\right\rangle\right| \leq K\left(\left\|C^{\frac{1}{2}} X_{0}\right\|^{2}+\left\|X_{0}\right\|^{2}\right)
$$

whenever $0 \leq s \leq T_{j} \leq \bar{s} \leq T_{\kappa}$. 
Proof. The linearity of (5.2) and the polarization identity allow us to assume without loss of generality that $B_{1}=B_{3}$ and $B_{2}=B_{4}$. It follows from (5.1) that

$$
\mathbf{E}\left\langle B Y_{T_{j}, n}^{s, B_{1} Z_{s, n}+B_{2} Z_{T_{j}, n}}, \Phi_{T_{\kappa}-\bar{s}, n} B Y_{T_{j}, n}^{s, B_{3} Z_{s, n}+B_{4} Z_{T_{j}, n}}\right\rangle=\mathbf{E}\langle\tilde{Y}, A \tilde{Y}\rangle,
$$

with $\tilde{Y}=Y_{T_{\kappa}, n}^{\bar{s}, B Y_{T_{j}, n}^{s, B_{1} Z_{s, n}+B_{2} Z_{T_{j}, n}}}$. Hence proceeding as in the proof of Lemma 5.3 we obtain

$$
\begin{aligned}
\left|\mathbf{E}\left(\langle\tilde{Y}, A \tilde{Y}\rangle / F_{\bar{s}}\right)\right| & \leq K\left(\left\|C_{2}^{\frac{1}{2}} B Y_{T_{j}, n}^{s, B_{1} Z_{s, n}+B_{2} Z_{T_{j}, n}}\right\|^{2}+\left\|B Y_{T_{j}, n}^{s, B_{1} Z_{s, n}+B_{2} Z_{T_{j}, n}}\right\|^{2}\right) \\
& \leq K\left(\left\|C_{1}^{\frac{1}{2}} Y_{T_{j}, n}^{s, B_{1} Z_{s, n}+B_{2} Z_{T_{j}, n}}\right\|^{2}+\left\|Y_{T_{j}, n}^{s, B_{1} Z_{s, n}+B_{2} Z_{T_{j}, n}}\right\|^{2}\right) .
\end{aligned}
$$

Therefore

$$
\begin{aligned}
\left|\mathbf{E}\left(\langle\tilde{Y}, A \tilde{Y}\rangle / F_{s}\right)\right| & \leq K\left(\left\|C_{1}^{\frac{1}{2}}\left(B_{1} Z_{s, n}+B_{2} Z_{T_{j}, n}\right)\right\|^{2}+\left\|B_{1} Z_{s, n}+B_{2} Z_{T_{j}, n}\right\|^{2}\right) \\
& \leq K\left(\left\|C^{\frac{1}{2}} Z_{s, n}\right\|^{2}+\left\|C^{\frac{1}{2}} Z_{T_{j}, n}\right\|^{2}+\left\|Z_{s, n}\right\|^{2}+\left\|Z_{T_{j}, n}\right\|^{2}\right) .
\end{aligned}
$$

Thus the desired result follows from the estimates given in the first part of the proof of Lemma 5.3.

Proof of Theorem 5.4. Returning to the proof of Theorem 5.1 one may notice that the polarization identity leads to

$$
\delta_{k}=-\frac{T}{2 M} \sum_{\left(B_{1}, B_{2}, 1\right) \in \mathcal{S}} \int_{T_{k}}^{T_{k+1}} \mathbf{E}\left\langle B_{1} X_{s, n}, \Phi_{T_{j}-s, n} B_{2} X_{s, n}\right\rangle d s-\tilde{\delta}_{k}^{1}-\tilde{\delta}_{k}^{2},
$$

with

$$
\begin{aligned}
\tilde{\delta}_{k}^{1}= & \sum_{(B, \bar{k}, r) \in \widetilde{\mathcal{S}}} i^{-\bar{k}} \int_{T_{k}}^{T_{k+1}} \mathbf{E}\left\langle B Z_{T_{k}, n}, \Phi_{T_{j}-s, n} B Z_{T_{k}, n}\right\rangle\left(s-T_{k}\right)^{r} d s \\
& -\sum_{(B, \bar{k}, r) \in \widetilde{\mathcal{S}}} i^{-\bar{k}} \int_{T_{k}}^{T_{k+1}} \mathbf{E}\left\langle B X_{T_{k}, n}, \Phi_{T_{j}-s, n} B X_{T_{k}, n}\right\rangle\left(s-T_{k}\right)^{r} d s, \\
\tilde{\delta}_{k}^{2}= & \sum_{(B, \bar{k}, r) \in \tilde{\mathcal{S}}} i^{-\bar{k}} \int_{T_{k}}^{T_{k+1}} \mathbf{E}\left\langle B X_{T_{k}, n}, \Phi_{T_{j}-s, n} B X_{T_{k}, n}\right\rangle\left(s-T_{k}\right)^{r} d s \\
& -\sum_{(B, \bar{k}, 1) \in \tilde{\mathcal{S}}} i^{-\bar{k}} \frac{T}{2 M} \int_{T_{k}}^{T_{k+1}} \mathbf{E}\left\langle B X_{s, n}, \Phi_{T_{j}-s, n} B X_{s, n}\right\rangle d s,
\end{aligned}
$$

where $\widetilde{\mathcal{S}}=\left\{(B, \bar{k}, r): \bar{k}=0, \ldots, 3, B=B_{1}+i^{\bar{k}} B_{2}\right.$ with $\left.\left(B_{1}, B_{2}, r\right) \in \mathcal{S}\right\}$.

We now focus on the estimation of $\tilde{\delta}_{k}^{1}$. For any $B$ present in (5.6), let us define the function $v_{n}$ by

$$
v_{n}(s, x, y)=\mathbf{E}\left\langle B Y_{T_{k}, n}^{s, \bar{x}}, \Phi_{T_{j}-\bar{s}, n} B Y_{T_{k}, n}^{s, y}\right\rangle
$$


provided that $0 \leq s \leq T_{k} \leq \bar{s} \leq T_{j}$ and $x, y \in \mathfrak{h}_{n}$. Thus for any $\bar{s} \in\left[T_{k}, T_{k+1}\right]$

$$
\begin{aligned}
& \mathbf{E}\left\langle B Z_{T_{k}, n}, \Phi_{T_{j}-\bar{s}, n} B Z_{T_{k}, n}\right\rangle-\mathbf{E}\left\langle B X_{T_{k}, n}, \Phi_{T_{j}-\bar{s}, n} B X_{T_{k}, n}\right\rangle \\
& \quad=\sum_{l=0}^{k-1} \mathbf{E} v_{n}\left(T_{l+1}, \overline{Z_{T_{l+1}, n}}, Z_{T_{l+1}, n}\right)-\mathbf{E} v_{n}\left(T_{l}, \overline{Z_{T_{l}, n}}, Z_{T_{l}, n}\right) .
\end{aligned}
$$

It follows from Itô's formula that

$$
\begin{aligned}
& \mathbf{E}\left(v_{n}\left(T_{l+1}, \overline{Z_{T_{l+1}, n}}, Z_{T_{l+1}, n}\right)-v_{n}\left(T_{l}, \overline{Z_{T_{l}, n}}, Z_{T_{l}, n}\right)\right) \\
& \quad=\mathbf{E} \int_{T_{l}}^{T_{l+1}}\left(\frac{\partial}{\partial r} v_{n}\left(r, \overline{Z_{r, n}}, Z_{r, n}\right)+\mathcal{L}_{\overline{Z_{T_{l}, n}}, Z_{T_{l}, n}}\left(v_{n}\right)\left(r, \overline{Z_{r, n}}, Z_{r, n}\right)\right) d r,
\end{aligned}
$$

with

$$
\begin{aligned}
\mathcal{L}_{\alpha, \beta}\left(v_{n}\right)(s, x, y)= & \sum_{l \in[\overrightarrow{0}, \vec{n}]} \frac{\partial v_{n}}{\partial x^{l}}(s, x, y)\left(\overline{G_{n}} \alpha\right)^{l}+\sum_{l \in[\overrightarrow{0}, \vec{n}]} \frac{\partial v_{n}}{\partial y^{l}}(s, x, y)\left(G_{n} \beta\right)^{l} \\
& +\frac{1}{2} \sum_{l=1}^{m} \sum_{\bar{k}, \bar{j} \in[\overrightarrow{0}, \vec{n}]} \frac{\partial^{2} v_{n}}{\partial x^{\bar{k}} x^{\bar{j}}}(s, x, y)\left(\overline{L_{l, n}} \alpha\right)^{\bar{k}}\left(\overline{L_{l, n}} \alpha\right)^{\bar{j}} \\
& +\frac{1}{2} \sum_{l=1}^{m} \sum_{\bar{k}, \bar{j} \in[\overrightarrow{0}, \vec{n}]} \frac{\partial^{2} v_{n}}{\partial x^{\bar{k}} y^{\bar{j}}}(s, x, y)\left(\overline{L_{l, n}} \alpha\right)^{\bar{k}}\left(L_{l, n} \beta\right)^{\bar{j}} \\
& +\frac{1}{2} \sum_{l=1}^{m} \sum_{\bar{k}, \bar{j} \in[\overrightarrow{0}, \vec{n}]} \frac{\partial^{2} v_{n}}{\partial y^{\bar{k}} x^{\bar{j}}}(s, x, y)\left(L_{l, n} \beta\right)^{\bar{k}}\left(\overline{L_{l, n}} \alpha\right)^{\bar{j}} \\
& +\frac{1}{2} \sum_{l=1}^{m} \sum_{\bar{k}, \bar{j} \in[\overrightarrow{0}, \vec{n}]} \frac{\partial^{2} v_{n}}{\partial y^{\bar{k}} y^{\bar{j}}}(s, x, y)\left(L_{l, n} \beta\right)^{\bar{k}}\left(L_{l, n} \beta\right)^{\bar{j}}
\end{aligned}
$$

where for any $O \in B\left(\mathfrak{h}_{n}\right)$, the operator $\bar{O}$ is defined by $\bar{O} \varphi_{k}=\overline{O \varphi_{k}}, k \in[\overrightarrow{0}, \vec{n}]$. Furthermore, again using Itô's formula, we arrive at

$$
\begin{aligned}
\mathbf{E}\left(v_{n}\right. & \left.\left(T_{l+1}, \overline{Z_{T_{l+1}, n}}, Z_{T_{l+1}, n}\right)-v_{n}\left(T_{l}, \overline{Z_{T_{l}, n}}, Z_{T_{l}, n}\right)\right) \\
= & \mathbf{E} \int_{T_{l}}^{T_{l+1}} \int_{T_{l}}^{r}\left(\mathcal{L}_{\overline{Z_{T_{l}, n}}, Z_{T_{l}, n}}\left(\mathcal{L}_{\overline{Z_{T_{l}, n}}, Z_{T_{l}, n}}\left(v_{n}\right)\right)\left(s, \overline{Z_{s, n}}, Z_{s, n}\right)\right) d s d r \\
& -2 \mathbf{E} \int_{T_{l}}^{T_{l+1}} \int_{T_{l}}^{r}\left(\mathcal{L}_{\overline{Z_{T_{l}, n}}, Z_{T_{l}, n}}\left(\mathcal{L}\left(v_{n}\right)\right)\left(s, \overline{Z_{s, n}}, Z_{s, n}\right)\right) d s d r \\
& +\mathbf{E} \int_{T_{l}}^{T_{l+1}} \int_{T_{l}}^{r}\left(\mathcal{L}\left(\mathcal{L}\left(v_{n}\right)\right)\left(s, \overline{Z_{s, n}}, Z_{s, n}\right)\right) d s d r,
\end{aligned}
$$

with $\mathcal{L}\left(v_{n}\right)(s, x, y)=\mathcal{L}_{x, y}\left(v_{n}\right)(s, x, y)$.

Now, a long calculation leads to

$$
\mathcal{L}_{\bar{\alpha}, \alpha}\left(\mathcal{L}_{\bar{\alpha}, \alpha}\left(v_{n}\right)\right)(s, \bar{x}, x)=2 \mathbf{E}\left\langle B Y_{T_{k}, n}^{s, G \alpha}, \Phi_{T_{\kappa}-\bar{s}, n} B Y_{T_{k}, n}^{s, G \alpha}\right\rangle
$$

and $\mathcal{L}_{\bar{\alpha}, \alpha}\left(\mathcal{L}\left(v_{n}\right)\right)(s, \bar{x}, x)=\sum_{(y, z) \in \mathcal{I}} \mathbf{E}\left\langle B Y_{T_{k}, n}^{s, y}, \Phi_{T_{\kappa}-\bar{s}, n} B Y_{T_{k}, n}^{s, z}\right\rangle$, where $\mathcal{I}$ is the set formed by the following pairs: $\left(G_{n}^{2} \alpha, x\right),\left(G_{n} \alpha, G_{n} x\right),\left(G_{n} x, G_{n} \alpha\right),\left(x, G_{n}^{2} \alpha\right)$, 
$\left(L_{l, n} G_{n} \alpha, L_{l, n} x\right),\left(L_{l, n} x, L_{l, n} G_{n} \alpha\right),\left(L_{l, n} \alpha, G_{n} L_{l, n} \alpha\right),\left(L_{l_{1}, n} L_{l, n} \alpha, L_{l_{1}, n} L_{l, n} \alpha\right)$ and $\left(G_{n} L_{l, n} \alpha, L_{l, n} \alpha\right)$ with $l, l_{1}=1, \ldots, m$. Therefore, Lemma 5.5 yields

$$
\left|\tilde{\delta}_{k}^{1}\right| \leq K\left(\frac{T}{M}\right)^{3}\left(\left\|C^{\frac{1}{2}} X_{0}\right\|^{2}+\left\|X_{0}\right\|^{2}\right) .
$$

It remains to estimate $\tilde{\delta}_{k}^{2}$. Combining the integration by parts formula and (5.3), we have that

$$
\begin{array}{rl}
\int_{T_{k}}^{T_{k+1}} & \mathbf{E}\left\langle B X_{T_{k}, n}, \Phi_{T_{j}-s, n} B X_{T_{k}, n}\right\rangle\left(s-T_{k}\right) d s \\
= & \frac{1}{2}\left(\frac{T}{M}\right)^{2} \mathbf{E}\left\langle B X_{T_{k}, n}, \Phi_{T_{j}-T_{k+1}, n} B X_{T_{k}, n}\right\rangle \\
& +\frac{1}{2} \int_{T_{k}}^{T_{k+1}} \mathbf{E}\left\langle G_{n} B X_{T_{k}, n}, \Phi_{T_{j}-s, n} B X_{T_{k}, n}\right\rangle\left(s-T_{k}\right)^{2} d s \\
& +\frac{1}{2} \int_{T_{k}}^{T_{k+1}} \mathbf{E}\left\langle B X_{T_{k}, n}, \Phi_{T_{j}-s, n} G_{n} B X_{T_{k}, n}\right\rangle\left(s-T_{k}\right)^{2} d s \\
& +\frac{1}{2} \sum_{l=1}^{m} \int_{T_{k}}^{T_{k+1}} \mathbf{E}\left\langle L_{l, n} B X_{T_{k}, n}, \Phi_{T_{j}-s, n} L_{l, n} B X_{T_{k}, n}\right\rangle\left(s-T_{k}\right)^{2} d s,
\end{array}
$$

where $(B, \bar{k}, 1) \in \widetilde{\mathcal{S}}$ for some $\bar{k}$. Since

$$
\begin{aligned}
\mathbf{E}\left\langle B X_{s, n}, \Phi_{T_{j}-s, n} B X_{s, n}\right\rangle= & \mathbf{E}\left\langle B X_{T_{k}, n}, \Phi_{T_{j}-s, n} B X_{T_{k}, n}\right\rangle \\
& +\int_{T_{k}}^{s} \mathbf{E}\left\langle B G_{n} X_{r, n}, \Phi_{T_{j}-s, n} B X_{r, n}\right\rangle d r \\
& +\int_{T_{k}}^{s} \mathbf{E}\left\langle B X_{r, n}, \Phi_{T_{j}-s, n} B G_{n} X_{r, n}\right\rangle d r \\
& +\sum_{l=1}^{m} \int_{T_{k}}^{s} \mathbf{E}\left\langle B L_{l, n} X_{r, n}, \Phi_{T_{j}-s, n} B L_{l, n} X_{r, n}\right\rangle d r,
\end{aligned}
$$

we conclude the proof by applying the integration by parts formula and (5.3) to $\int_{T_{k}}^{T_{k+1}} \mathbf{E}\left\langle B X_{T_{k}, n}, \Phi_{T_{j}-s, n} B X_{T_{k}, n}\right\rangle d s$ and arguing similarly as in the last part of the proof of Lemma 5.3

\section{Application}

This section is devoted to illustrating the main results of this paper. More precisely, in view of the harmonic oscillators' considerable importance in quantum mechanics [4, 21, we develop here an example of a forced and damped quantum harmonic oscillator in the interaction representation. Then this concrete situation allows us to give the flavor of the hypotheses that support our presentation. We begin by establishing the corresponding notation.

In what follows, we assume $\mathfrak{h}=l^{2}\left(\mathbb{Z}_{+}\right)$and that $\left(\varphi_{k}\right)_{k \geq 0}$ is the canonical orthonormal basis on the space $l^{2}\left(\mathbb{Z}_{+}\right)$. Moreover, we denote by $a^{\dagger}$, $a$ the creation and annihilation operators; that is, the domain of $a^{\dagger}, a$ is

$$
\left\{x \in l^{2}\left(\mathbb{Z}_{+}\right): \sum_{k \geq 0} k\left|x_{k}\right|^{2}<+\infty\right\}
$$


and $a^{\dagger} \varphi_{n}=\sqrt{n+1} \varphi_{n+1}$,

$$
a \varphi_{n}= \begin{cases}0, & \text { if } n=0 \\ \sqrt{n} \varphi_{n-1}, & \text { if } n>0\end{cases}
$$

The number operator is given by $N=a^{\dagger} a$.

From now on we consider the system defined by the effective Hamiltonian

$$
H=i k_{1}\left(a^{\dagger}-a\right)+k_{2} N
$$

and the Lindblad operators $L_{1}=c_{1} a, L_{2}=c_{2} a^{2}, L_{3}=c_{3} N, L_{4}=c_{4} a^{\dagger}$, where $k_{1}, k_{2} \in \mathbb{R}$ and $c_{1}, \ldots, c_{4} \in \mathbb{C}$.

The model presented here describes many physical phenomena, for instance a single mode of a quantized electromagnetic field. In this framework, the vectors $\varphi_{k}$, $k=0,1, \ldots$, characterize the levels of energy. Moreover, the operator $a^{\dagger}$ creates a photon, whereas the operator $a$ destroys a photon. Then the term $i k_{1}\left(a^{\dagger}-a\right)$ describes a driving force or lineal pumping and $L_{1}$ describes the damping due to photon emission. $\mathbf{E}\left\langle X_{t}, N X_{t}\right\rangle$ is the mean photon number or energy at time $t$.

Let $\mathfrak{h}_{n}$ be equal to the linear manifold spanned by $\left\{\varphi_{j}: 0 \leq j \leq n\right\}$. Then $\mathfrak{D}^{*}=$ $\bigcup_{n=0}^{\infty} \mathfrak{h}_{n}$ satisfies hypothesis H4. In addition, for any $\phi=\left(\phi_{0}, \ldots, \phi_{n}, 0, \ldots\right) \in \mathfrak{h}_{n}$,

$$
\begin{aligned}
& 2 \operatorname{Re}\left\langle N^{p} \phi, N^{p} G_{n} \phi\right\rangle+\sum_{k=1}^{m}\left\|N^{p} L_{k, n} \phi\right\|^{2} \\
& =\sum_{j=1}^{n}\left|c_{1}\right|^{2}\left|\phi_{j}\right|^{2} j\left(-j^{2 p}+(j-1)^{2 p}\right)+\left|c_{2}\right|^{2}\left|\phi_{j}\right|^{2} j(j-1)\left(-j^{2 p}+(j-2)^{2 p}\right) \\
& \quad+\sum_{j=0}^{n-1}\left|c_{4}\right|^{2}\left|\phi_{j}\right|^{2}(j+1)\left(-j^{2 p}+(j+1)^{2 p}\right) \\
& \quad+2 k_{1} \sqrt{j+1} \operatorname{Re}\left(\phi_{j+1} \overline{\phi_{j}}\right)\left((j+1)^{2 p}-j^{2 p}\right) .
\end{aligned}
$$

Hence H7 is fulfilled by any $C=N^{2 p}$, with $p \in \mathbb{Z}_{+}$. Since for any $\phi \in \operatorname{Dom}(G)$, $\operatorname{Re}\langle\phi, G \phi\rangle=-\frac{1}{2} \sum_{k=1}^{m}\left\|L_{k} \phi\right\|^{2} \leq 0, G$ is a dissipative linear operator on $\mathfrak{h}$. Furthermore, $\left\|G P_{n}-P_{n} G P_{n}\right\| \leq\left|k_{1}\right| \sqrt{n+1}+\frac{\left|c_{4}\right|^{2}}{2}(n+1)$. Thus H1 follows from Theorem 2 of [11]. Notice that $G \in O_{2,1,1}$ and $L_{j} \in O_{1,2,1}, j=1, \ldots, 4$. Therefore, Theorem 4.2 implies

Proposition 6.1. Let $A \in O_{l_{A}, n_{A}, m_{A}}$ and $p \in \mathbb{N}$ such that $p \geq \max \left\{l_{A}, 2\right\}$. If $X_{0} \in \operatorname{Dom}\left(N^{p}\right)$, then for any $n>0$,

$$
\begin{aligned}
& \sup _{t \in[0, T]}\left|\mathbf{E}\left\langle X_{t}, A X_{t}\right\rangle-\mathbf{E}\left\langle X_{t, n}, A X_{t, n}\right\rangle\right| \\
& \quad \leq K\left(\left\|N^{p} X_{0}\right\|^{2}+\left\|X_{0}\right\|^{2}\right)\left(\frac{1}{n^{p-2}}+\frac{1}{n^{2 p-4-l_{A}}}\right) .
\end{aligned}
$$


Furthermore, for any $\phi \in \mathfrak{h}_{n}$ a straightforward computation yields that $\left\|N^{p} G_{n} \phi\right\|^{2}$ is equal to

$$
\begin{aligned}
\left(k_{1}\left|\phi_{n-1}\right|\right)^{2} n^{2 p+1}+n^{2 p}\left|\phi_{n}\right|^{2}\left|i k_{2} n+\frac{\left|c_{1}\right|^{2} n}{2}+\frac{\left|c_{2}\right|^{2} n(n-1)}{2}+\frac{\left|c_{3}\right|^{2} n^{2}}{2}\right|^{2} \\
+\sum_{j=1}^{n-1} j^{2 p} \mid k_{1} \phi_{j-1} \sqrt{j}-k_{1} \phi_{j+1} \sqrt{j+1} \\
\quad-\left.\phi_{j}\left(i k_{2} j+\frac{\left|c_{1}\right|^{2} j}{2}+\frac{\left|c_{2}\right|^{2} j(j-1)}{2}+\frac{\left|c_{3}\right|^{2} j^{2}}{2}+\frac{\left|c_{4}\right|^{2}(j+1)}{2}\right)\right|^{2} .
\end{aligned}
$$

Thus choosing $c_{n}=n^{4}$, we can obtain condition S2. Then Proposition 6.2 follows from Theorem 5.1

Proposition 6.2. Let $A \in O_{l_{A}, n_{A}, m_{A}}$ and $p \in \mathbb{N}$ such that $p \geq \max \left\{l_{A} / 2+4, l_{A}\right\}$. If there exists a constant $\mu$ such that $n^{4} / M \leq \mu$, then

$$
\begin{aligned}
& \left|\mathbf{E}\left\langle X_{T_{j}}, A X_{T_{j}}\right\rangle-\mathbf{E}\left\langle Z_{T_{j}, n}^{M}, A Z_{T_{j}, n}^{M}\right\rangle\right| \\
& \quad \leq K_{p}\left(\left\|N^{p} X_{0}\right\|^{2}+\left\|X_{0}\right\|^{2}\right)\left(\frac{T}{M}+\frac{1}{n^{p-2}}+\frac{1}{n^{2 p-4-l_{A}}}\right),
\end{aligned}
$$

where the constant $K_{p}$ depends on $p$. Furthermore, for any $p \geq\left\{l_{A} / 2+8, l_{A}\right\}$,

$$
\begin{gathered}
\left|\mathbf{E}\left\langle X_{T_{j}^{M}}, A X_{T_{j}^{M}}\right\rangle-2 \mathbf{E}\left\langle Z_{T_{2 j}^{2 M}, n}^{2 M}, A Z_{T_{2 j}^{2 M}, n}^{2 M}\right\rangle+\mathbf{E}\left\langle Z_{T_{j}^{M}, n}^{M}, A Z_{T_{j}^{M}, n}^{M}\right\rangle\right| \\
\leq K_{p}\left(\left\|N^{p} X_{0}\right\|^{2}+\left\|X_{0}\right\|^{2}\right)\left(\left(\frac{T}{M}\right)^{2}+\frac{1}{n^{p-2}}+\frac{1}{n^{2 p-4-l_{A}}}\right) .
\end{gathered}
$$

\section{ACKNOWLEDGMENTS}

I am indebted to Prof. R. Rebolledo for his valuable advice. I thank Prof. F. Fagnola, Prof. C. Saavedra and Prof. D. Talay for many helpful comments and suggestions. I also thank the anonymous referee and editor for their helpful comments. I acknowledge the warm hospitality of the "Facultad de Matemáticas, Pontificia Universidad Católica de Chile", where a big part of this research was performed.

\section{REFERENCES}

[1] R. Balescu, Equilibrium and nonequilibrium statistical mechanics. Krieger Publishing Company (1991) (original edition 1975). MR 53:12405

[2] A. Barchielli, A.M. Paganoni, F. Zucca, On stochastic differential equations and semigroups of probability operators in quantum probability. Stochastic Proc. and their Appl. 73 (1998), 69-86. MR 99k:81138

[3] H. Carmichael, An open systems approach to quantum optics. Lecture Notes in Physics. Springer-Verlag (1993).

[4] C. Cohen-Tannoudji, B. Diu, F. Laloë, Quantum mechanics. Volume 1. Hermann (1977).

[5] A.M. Chebotarev, F. Fagnola, Sufficient conditions for conservativity of minimal quantum dynamical semigroups. J. Funct. Anal. 153 (1998), 382-404. MR 99d:81064

[6] F. Fagnola, R. Rebolledo, C. Saavedra, Quantum flows associated to master equations in quantum optics. J. Math. Phys. 35 (1994), 1-12. MR 94k:81353

[7] F. Fagnola, Quantum markov semigroups and quantum flows. Proyecciones 18 (1999), 1-144. MR 2002f: 81054 
[8] F. Fagnola, S. J. Wills, Solving quantum stochastic differential equations with unbounded coefficients. J. Funct. Anal. 198 (2003), 279-310.

[9] N. Gisin, I.C. Percival, The quantum-state diffusion model applied to open systems. J. Phys. A 25 (1992), 5677-5691. MR 94b:82028

[10] A.S. Holevo, On dissipative stochastic equations in a Hilbert space. Probab. Theory Related Fields 104 (1996), 483-500. MR 98c:60068

[11] P.T. Jorgensen, Approximately reducing subspaces for unbounded linear operators. J. Funct. Anal. 23 (1976), 392-414. MR 98c:60068

[12] T. Kato, Perturbation theory for linear operators. Corrected printing of the second edition. Springer-Verlag (1980). MR 96a:47025

[13] P.E. Kloeden, E. Platen, Numerical solution of stochastic differential equations. Springer, Berlin (1992). MR 94b:60069

[14] Tarso B.L. Kist, M. Orszag, T.A. Brun, L. Davidovich, Stochastic Schrödinger equations in cavity QDE: physical interpretation and localization. J. Opt. B: Quantum Semiclass. Opt. 1 (1999), 251-263.

[15] R. Dautray, J.-L. Lions, Mathematical analysis and numerical methods for science and technology. Vol. 5, Evolution Problems I, Springer-Verlag (1992). MR 92k:00006

[16] G.N. Milstein, The numerical integration of stochastic differentials equations. Ural University Press (1988).

[17] C. Mora, Solución numérica de ecuaciones diferenciales estocásticas mediante métodos exponenciales. Ph.D. Thesis, Pontificia Universidad Católica de Chile (2002).

[18] A. Pazy, Semigroups of linear operators and applications to partial differential equations. Springer-Verlag (1975). MR 85g:47061

[19] I.C. Percival, Quantum state diffusion. Cambridge University Press (1998). MR 2000f:81023

[20] P. Protter, Stochastic integration and differential equations. Springer (1990). MR 91i:60148

[21] M.O. Scully, M.S. Zubairy, Quantum optics. Cambridge University Press (1997).

[22] W. T. Strunz, L. Diósi, N. Gisin, T. Yu, Quantum trajectories for Brownian motion. Phys. Rev. Lett. 83 (1999), 4909-4913. MR 2000h:82042

[23] D. Talay, Efficient numerical schemes for the approximation of expectations of functionals of the solution of an SDE and applications. Springer Lecture Notes in Control and Inform. Sc. 61 (1984), 294-313. MR 88b:60140

[24] D. Talay, Discrétisation d'une E.D.S. et calcul approaché d'espérances de fonctionnelles de la solution. Math. Mod. Numer. Anal. 20 (1986), 141-179. MR 87k:60153

[25] D. Talay, L. Tubaro, Expansion of the global error for numerical schemes solving stochastic differential equations. Stochastic Anal. Appl. 8 (1990), 483-509. MR 92e:60124

[26] D. Talay, Stochastic Hamiltonian systems: exponential convergence to the invariant measure, and discretization by the implicit Euler scheme. Markov Processes. Related Fields 8 (2002), 163-198. MR 2003e:60129]

Departamento de Ingeniería Matemática, Universidad de Concepción, Concepción, CHILE

Current address: Departamento de Ingeniería Matemática, Universidad de Concepción, Casilla 160 C, Concepción, Chile

E-mail address: cmora@ing-mat.udec.cl 